Acta Crystallographica Section C

Structural Chemistry

ISSN 2053-2296

\section{Three-dimensional hydrogen-bonded framework structures in flunarizinium nicotinate and flunarizinediium bis(4-toluenesulfonate) dihydrate}

\author{
Channappa N. Kavitha, ${ }^{a}$ Hemmige S. Yathirajan, ${ }^{a}$ \\ Manpreet Kaur, ${ }^{a}$ Eric C. Hosten, ${ }^{b}$ Richard Betz ${ }^{b}$ and \\ Christopher Glidewell ${ }^{\mathrm{C} *}$
}

a University of Mysore, Department of Studies in Chemistry, Manasagangotri, Mysore 570 006, India, 'b Department of Chemistry, Nelson Mandela Metropolitan University, Summerstrand Campus (South), University Way, Summerstrand, PO Box 77000, Port Elizabeth 6031, South Africa, and ' School of Chemistry, University of St Andrews, Fife KY16 9ST, Scotland

Correspondence e-mail:cg@st-andrews.ac.uk

Received 15 July 2014

Accepted 16 July 2014

The structures of two salts of flunarizine, namely 1-bis[(4fluorophenyl)methyl]-4-[(2E)-3-phenylprop-2-en-1-yl]piperazine, $\mathrm{C}_{26} \mathrm{H}_{26} \mathrm{~F}_{2} \mathrm{~N}_{2}$, are reported. In flunarizinium nicotinate \{systematic name: 4-bis[(4-fluorophenyl)methyl]-1-[(2E)-3phenylprop-2-en-1-yl]piperazin-1-ium pyridine-3-carboxylate\}, $\mathrm{C}_{26} \mathrm{H}_{27} \mathrm{~F}_{2} \mathrm{~N}_{2}{ }^{+} \cdot \mathrm{C}_{6} \mathrm{H}_{4} \mathrm{NO}_{2}{ }^{-}$, (I), the two ionic components are linked by a short charge-assisted $\mathrm{N}-\mathrm{H} \cdots \mathrm{O}$ hydrogen bond. The ion pairs are linked into a three-dimensional framework structure by three independent $\mathrm{C}-\mathrm{H} \cdots \mathrm{O}$ hydrogen bonds, augmented by $\mathrm{C}-\mathrm{H} \cdots \pi$ (arene) hydrogen bonds and an aromatic $\pi-\pi$ stacking interaction. In flunarizinediium bis(4-toluenesulfonate) dihydrate \{systematic name: 1-[bis(4-fluorophenyl)methyl]-4-[(2E)-3-phenylprop-2en-1-yl]piperazine-1,4-diium bis(4-methylbenzenesulfonate) dihydrate, $\mathrm{C}_{26} \mathrm{H}_{28} \mathrm{~F}_{2} \mathrm{~N}_{2}{ }^{2+} \cdot 2 \mathrm{C}_{7} \mathrm{H}_{7} \mathrm{O}_{3} \mathrm{~S}^{-} \cdot 2 \mathrm{H}_{2} \mathrm{O}$, (II), one of the anions is disordered over two sites with occupancies of 0.832 (6) and 0.168 (6). The five independent components are linked into ribbons by two independent $\mathrm{N}-\mathrm{H}$. . O hydrogen bonds and four independent $\mathrm{O}-\mathrm{H}$. . O hydrogen bonds, and these ribbons are linked to form a three-dimensional framework by two independent $\mathrm{C}-\mathrm{H}$. . O hydrogen bonds, but $\mathrm{C}-\mathrm{H} \cdots \pi$ (arene) hydrogen bonds and aromatic $\pi-\pi$ stacking interactions are absent from the structure of (II). Comparisons are made with some related structures.

Keywords: crystal structure; flunarizinediium salt; flunarizinium salt; hydrogen bonding; $\pi-\pi$ stacking; calcium-channel blocker.

\section{Introduction}

Flunarizine \{systematic name: 1-[bis(4-fluorophenyl)methyl]4-[(2E)-3-phenylprop-2-en-1-yl]piperazine $\}$ is a nonselective calcium-channel blocker (Amery, 1983; Fagbemi et al., 1984; Tarland \& Flatmark, 1999) which is effective in the prophylaxis of migraine, occlusive peripheral vascular disease and vertigo of central and peripheral origin, and also as an adjuvant in the therapy of epilepsy. Its pharmacodynamic and pharmacokinetic properties and its therapeutic use have been reviewed (Holmes et al., 1984). Brief reports on the structures of several salts derived from flunarizine (Kavitha, Jasinski et al., 2013; Kavitha, Yathirajan et al., 2013; Shivaprakash et al., 2014) and from substituted piperazine derivatives closely related to flunarizine (Kavitha, Butcher et al., 2013; Kavitha, Yildirim et al., 2013) have been published recently, and we report here the molecular and supramolecular structures of flunarizinium nicotinate, (I) (Fig. 1), and flunarizinediium bis(4-toluenesulfonate) dihydrate, (II) (Fig. 2). The main purposes of the present study are, firstly, to compare the supramolecular assemblies of (I) and (II), and, secondly, to compare the structures of (I) and (II) with those of some closely related analogues.

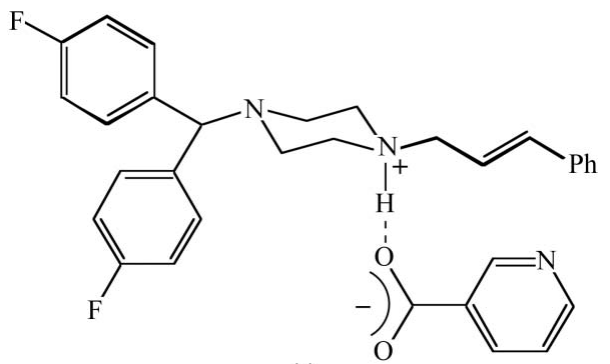

(I)

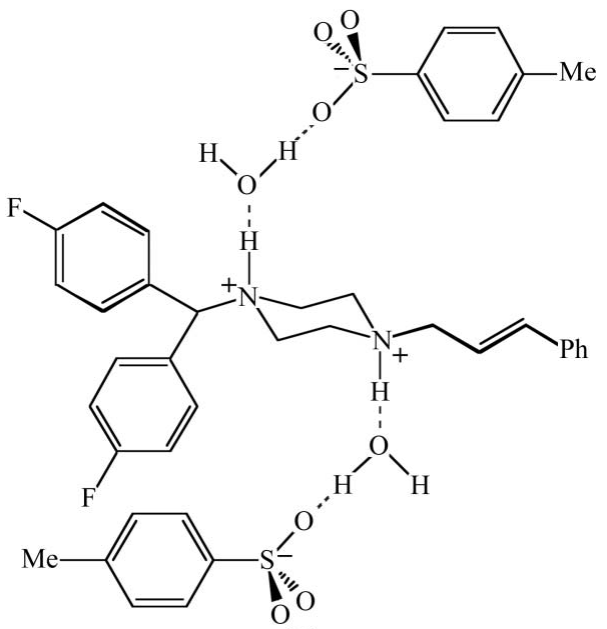

(II)

\section{Experimental}

\subsection{Synthesis and crystallization}

For the synthesis of salt (I), flunarizine free base $(4.05 \mathrm{~g}$, $0.01 \mathrm{~mol})$ and nicotinic acid $(1.23 \mathrm{~g}, 0.01 \mathrm{~mol})$ were dissolved in hot dimethylformamide $(5 \mathrm{ml})$ and the solution was stirred for $10 \mathrm{~min}$. The solution was then allowed to cool slowly to ambient temperature in the presence of air. Colourless crystals of (I) suitable for single-crystal X-ray diffraction appeared after a few days and were collected by filtration (m.p. 383- 
Table 1

Experimental details.

(I)

\section{Crystal data}

Chemical formula

$M_{\text {r }}$

Crystal system, space group

Temperature (K)

$a, b, c(\AA)$

$\beta\left({ }^{\circ}\right)$

$V\left(\AA^{3}\right)$

$Z$

Radiation type

$\mu\left(\mathrm{mm}^{-1}\right)$

Crystal size (mm)

Data collection

Diffractometer

Absorption correction

$T_{\min }, T_{\max }$

No. of measured, independent and observed

$[I>2 \sigma(I)]$ reflections

$R_{\mathrm{int}}$

$(\sin \theta / \lambda)_{\max }\left(\AA^{-1}\right)$

Refinement

$R\left[F^{2}>2 \sigma\left(F^{2}\right)\right], w R\left(F^{2}\right), S$

No. of reflections

No. of parameters

No. of restraints

$\mathrm{H}$-atom treatment

$\Delta \rho_{\max }, \Delta \rho_{\min }\left(\mathrm{e} \AA^{-3}\right)$

Absolute structure

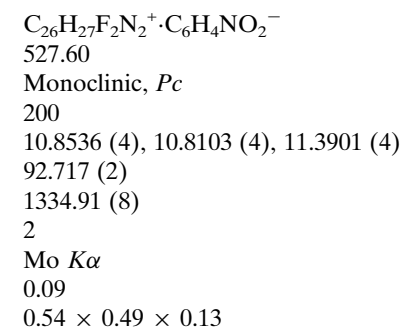

Monoclinic, $P c$

200

10.8536 (4), 10.8103 (4), 11.3901 (4)

$92.717(2)$

$1334.91(8)$

2

Mo $K \alpha$

0.09

$0.54 \times 0.49 \times 0.13$

Bruker APEXII CCD area-detector diffractometer Multi-scan (SADABS; Sheldrick, 2003) $0.873,0.988$

$23278,6092,5770$

\subsection{7}

0.668

\section{$0.030,0.081,1.03$}

6092

352

2

$\mathrm{H}$-atom parameters constrained

$0.15,-0.18$

Flack $x$ parameter (Flack, 1983) determined using 2539 quotients $\left[\left(I^{+}\right)-\left(I^{-}\right)\right] /\left[\left(I^{+}\right)+\left(I^{-}\right)\right]$(Parsons et al., 2013)

(II)

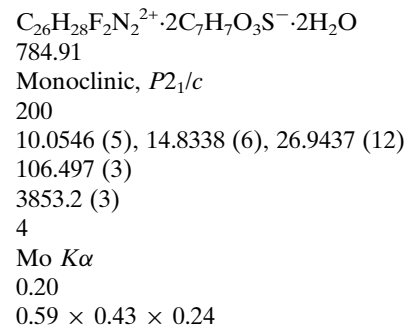

Bruker APEXII CCD area-detector diffractometer Multi-scan (SADABS; Sheldrick, 2003) 


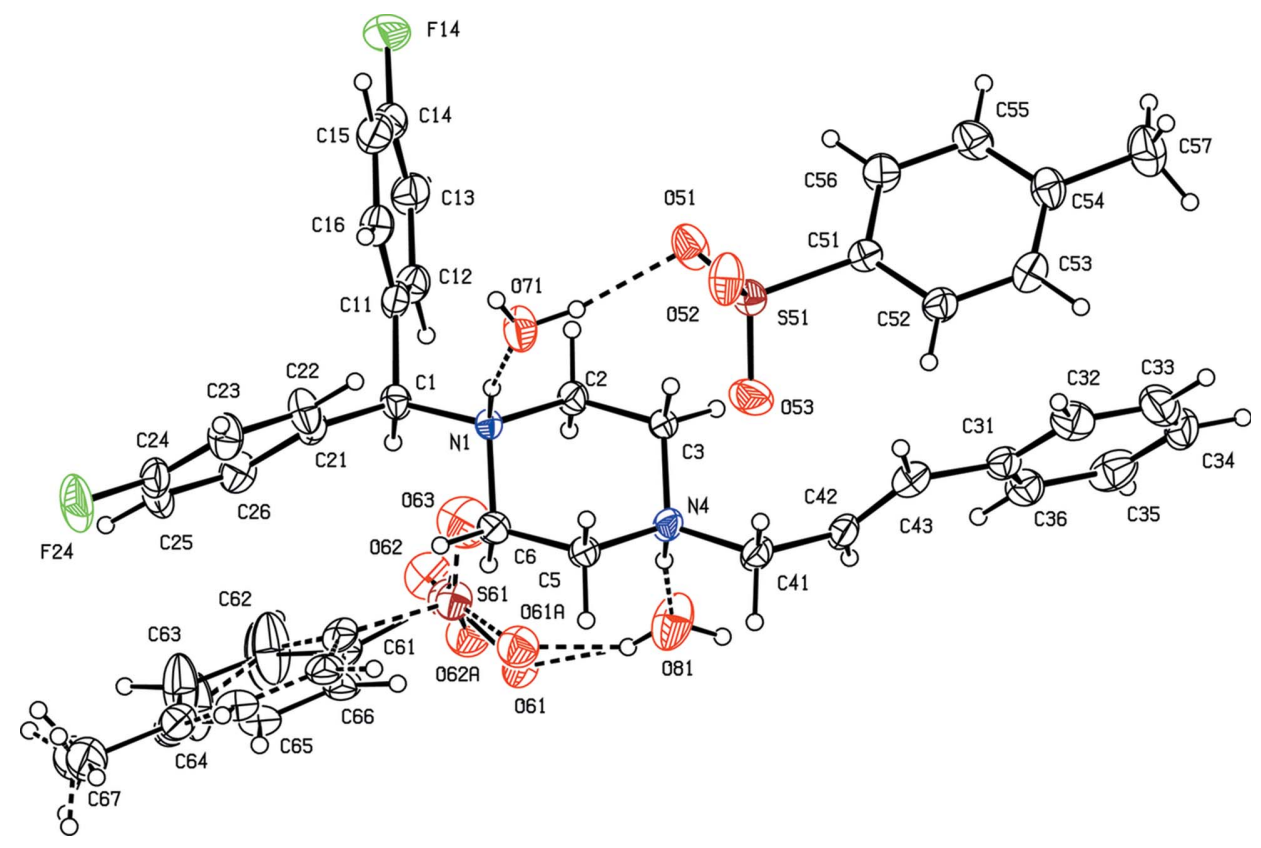

Figure 2

The independent components of hydrated salt (II), showing the atom-labelling scheme and the $\mathrm{O}-\mathrm{H} \cdots \mathrm{O}$ and $\mathrm{N}-\mathrm{H} \cdots \mathrm{O}$ hydrogen bonds (dashed lines) within the selected asymmetric unit. Displacement ellipsoids are drawn at the $30 \%$ probability level and the site occupancies for the two orientations of the disordered anion are 0.832 (6) and 0.168 (6). The pairs of atomic sites S61 and S61 A, and O63 and O63A, are nearly coincident and, for the sake of clarity, the atom labels $\mathrm{C} 61 A-\mathrm{C} 67 A, \mathrm{~S} 61 A$ and $\mathrm{O} 63 A$ have been omitted.

tals were all of very indifferent quality and about a dozen individual crystals were subjected to preliminary examination using polarizing microscopy; none showed any visible signs of twinning or intergrowth. Three data sets were obtained from the two most promising looking crystals and the refinement reported here results from the best of these data sets, although all three gave essentially identical solutions. For all three data sets, it was apparent from an early stage that one of the anions, that containing atom S61 (Fig. 2), was disordered over two sets of sites. For the minor orientation, the directly bonded distances and the one-angle distances were restrained to be equal to the corresponding distances in the major orientation, subject to standard uncertainties of 0.005 and $0.01 \AA$, respectively. In addition, the anisotropic displacement parameters for the corresponding pairs of partial-occupancy atoms which occupied approximately the same regions of physical

Table 2

Hydrogen-bond geometry $\left(\AA,^{\circ}\right)$ for (I).

$C g 1$ and $C g 2$ represent the centroids of the $\mathrm{C} 11-\mathrm{C} 16$ and $\mathrm{C} 31-\mathrm{C} 36$ rings, respectively.

\begin{tabular}{|c|c|c|c|c|}
\hline$D-\mathrm{H} \cdots A$ & $D-\mathrm{H}$ & $\mathrm{H} \cdots A$ & $D \cdots A$ & $D-\mathrm{H} \cdots A$ \\
\hline $\mathrm{N} 4-\mathrm{H} 4 \cdots \mathrm{O} 51$ & 0.98 & 1.58 & $2.5630(19)$ & 175 \\
\hline $\mathrm{C} 3-\mathrm{H} 3 B \cdots \mathrm{O} 52^{\mathrm{i}}$ & 0.99 & 2.53 & $3.440(2)$ & 153 \\
\hline $\mathrm{C} 13-\mathrm{H} 13 \cdots \mathrm{O} 52^{\mathrm{ii}}$ & 0.95 & 2.57 & $3.442(2)$ & 152 \\
\hline $\mathrm{C} 56-\mathrm{H} 56 \cdots \mathrm{O} 51^{\mathrm{iii}}$ & 0.95 & 2.34 & $3.236(3)$ & 158 \\
\hline $\mathrm{C} 26-\mathrm{H} 26 \cdots C g 1^{\text {iv }}$ & 0.95 & 2.81 & $3.758(2)$ & 173 \\
\hline $\mathrm{C} 55-\mathrm{H} 55 \cdots \mathrm{Cg} 2^{\mathrm{v}}$ & 0.95 & 2.85 & $3.567(2)$ & 133 \\
\hline
\end{tabular}

Symmetry codes: (i) $x,-y+1, z+\frac{1}{2}$; (ii) $x-1,-y+1, z+\frac{1}{2}$; (iii) $x,-y+2, z-\frac{1}{2}$; (iv) $x,-y+1, z-\frac{1}{2}$; (v) $x, y, z-1$. space were constrained to be identical. Subject to these conditions, the site occupancies for the two orientations refined to 0.832 (6) and 0.168 (6). Because of the rather weak diffraction at high angles, all reflections having $\theta>25.5^{\circ}$ were omitted from the final refinements for hydrated salt (II). Examination of the refined structures of (I) and (II) using PLATON (Spek, 2009) showed an absence of any solventaccessible voids for both structures. For the rerefinement of (VI) (see Results and discussion, §3), the reported unit cell in the space group $P 2_{1} / n$ (Kavitha, Butcher et al., 2013) was transformed to the $P 2_{1} / c$ setting with dimensions $a=$ 10.0845 (2) $\AA, b=14.6026$ (3) $\mathrm{\AA}, c=27.1907$ (7) $\AA$ and $\beta=$ $108.315(2)^{\circ}$.

Table 3

Hydrogen-bond geometry $\left(\AA,^{\circ}\right)$ for (II).

\begin{tabular}{|c|c|c|c|c|}
\hline$D-\mathrm{H} \cdots A$ & $D-\mathrm{H}$ & $\mathrm{H} \cdots A$ & $D \cdots A$ & $D-\mathrm{H} \cdots A$ \\
\hline $\mathrm{N} 1-\mathrm{H} 1 \cdots \mathrm{O} 71$ & 0.93 & 1.79 & $2.716(4)$ & 169 \\
\hline $\mathrm{N} 4-\mathrm{H} 4 \cdots \mathrm{O} 81$ & 1.00 & 1.69 & $2.690(4)$ & 173 \\
\hline $\mathrm{O} 71-\mathrm{H} 71 A \cdots \mathrm{O} 51$ & 0.85 & 1.88 & $2.708(4)$ & 167 \\
\hline $\mathrm{O} 71-\mathrm{H} 71 B \cdots \mathrm{O} 61^{\mathrm{i}}$ & 0.84 & 1.87 & $2.675(5)$ & 160 \\
\hline $\mathrm{O} 81-\mathrm{H} 81 A \cdots \mathrm{O} 61$ & 0.84 & 2.05 & $2.852(5)$ & 161 \\
\hline $\mathrm{O} 81-\mathrm{H} 81 B \cdots \mathrm{O} 52^{\mathrm{ii}}$ & 0.84 & 1.92 & $2.748(4)$ & 167 \\
\hline $\mathrm{C} 1-\mathrm{H} 1 A \cdots \mathrm{O} 63$ & 1.00 & 2.23 & $3.139(7)$ & 151 \\
\hline $\mathrm{C} 2-\mathrm{H} 2 B \cdots \mathrm{O} 63$ & 0.99 & 2.47 & $3.314(7)$ & 143 \\
\hline $\mathrm{C} 6-\mathrm{H} 6 A \cdots \mathrm{O} 53^{\mathrm{iii}}$ & 0.99 & 2.37 & $3.191(5)$ & 140 \\
\hline $\mathrm{C} 16-\mathrm{H} 16 \cdots \mathrm{O} 62^{\mathrm{i}}$ & 0.95 & 2.41 & $3.228(6)$ & 144 \\
\hline $\mathrm{C} 22-\mathrm{H} 22 \cdots \mathrm{O} 71$ & 0.95 & 2.49 & $3.381(5)$ & 156 \\
\hline $\mathrm{C} 34-\mathrm{H} 34 \cdots \mathrm{O} 51^{\mathrm{iv}}$ & 0.95 & 2.42 & $3.362(6)$ & 169 \\
\hline $\mathrm{C} 41-\mathrm{H} 41 A \cdots \mathrm{O} 53$ & 0.99 & 2.35 & $3.280(5)$ & 157 \\
\hline $\mathrm{C} 41-\mathrm{H} 41 B \cdots \mathrm{O} 61^{\mathrm{v}}$ & 0.99 & 2.37 & $3.341(5)$ & 167 \\
\hline
\end{tabular}

Symmetry codes: (i) $x+1, y, z$; (ii) $x-1, y, z$; (iii) $-x+2,-y+1,-z$; (iv) $-x+2, y+\frac{1}{2},-z+\frac{1}{2} ;(\mathrm{v})-x+1,-y+1,-z$. 


\section{Results and discussion}

Salt (I) consists of flunarizinium monocations, in which protonation of the flunarizine free base has occurred selectively at atom $\mathrm{N} 4$, which is the $\mathrm{N}$ atom more remote from the electronegative fluorophenyl units, and nicotinate anions; the asymmetric unit was selected such that the two ions within it are linked by an $\mathrm{N}-\mathrm{H} \cdots \mathrm{O}$ hydrogen bond (Fig. 1 and Table 2). The constitution of (II) is more complex, comprising a flunarizinediium dication, two 4-methylbenzenesulfonate anions, one of which exhibits positional disorder, and two water molecules. Accordingly, there is considerable flexibility in the choice of the asymmetric unit. However, it is possible to specify a reasonably compact asymmetric unit within which the five independent components are linked by $\mathrm{N}-\mathrm{H} \cdots \mathrm{O}$ and $\mathrm{O}-\mathrm{H} \cdots \mathrm{O}$ hydrogen bonds (Fig. 2 and Table 3 ).

In each of (I) and (II), the pyrazine ring adopts a chair conformation, with the two hydrocarbyl substituents occupying equatorial sites (Figs. 1 and 2). The ring-puckering parameters (Cremer \& Pople, 1975), calculated for the atom sequence N1-C2-C3-N4-C5-C6, are $Q=0.5934$ (17) Å, $\theta=$ $3.56(15)^{\circ}$ and $\varphi=345(3)^{\circ}$ for (I), and $Q=0.554$ (3) $\AA, \theta=$ $3.0(3)^{\circ}$ and $\varphi=326(8)^{\circ}$ for (II). For an ideal chair conformation, the value of $\theta$ is exactly zero (Boeyens, 1978).

The 3-phenylprop-2-en-1-yl substituents in (I) and (II) adopt very similar orientations relative to the piperazine ring and very similar overall conformations, as shown by the relevant torsion angles (Tables 4 and 5). On the other hand, the orientations of the two independent fluorophenyl rings are somewhat different in the two compounds. This may be ascribed, at least in part, to the different direction-specific interactions involving these rings in the two compounds, including the $\mathrm{C}-\mathrm{H} \cdots \mathrm{O}$ and $\mathrm{C}-\mathrm{H} \cdots \pi$ (arene) hydrogen bonds and the aromatic $\pi-\pi$ stacking interactions, which differ markedly between the two compounds, as discussed below. There is no internal symmetry in the cation in either compound, so that these cations are conformationally chiral. However, in each case, the presence of glide planes confirms that equal numbers of the two conformational enantiomers are present.

Within the selected asymmetric unit of (I) (Fig. 1), the two components are linked by a rather short and nearly linear charge-assisted (Gilli et al., 1994) N-H - O hydrogen bond

Table 4

Selected torsion angles $\left({ }^{\circ}\right)$ for $(\mathrm{I})$.

\begin{tabular}{lclr}
\hline $\mathrm{C} 2-\mathrm{N} 1-\mathrm{C} 1-\mathrm{C} 11$ & $-70.92(16)$ & $\mathrm{C} 3-\mathrm{N} 4-\mathrm{C} 41-\mathrm{C} 42$ & $-55.58(19)$ \\
$\mathrm{C} 2-\mathrm{N} 1-\mathrm{C} 1-\mathrm{C} 21$ & $165.22(13)$ & $\mathrm{N} 4-\mathrm{C} 41-\mathrm{C} 42-\mathrm{C} 43$ & $118.27(19)$ \\
$\mathrm{N} 1-\mathrm{C} 1-\mathrm{C} 11-\mathrm{C} 12$ & $150.17(15)$ & $\mathrm{C} 41-\mathrm{C} 42-\mathrm{C} 43-\mathrm{C} 31$ & $177.23(16)$ \\
$\mathrm{N} 1-\mathrm{C} 1-\mathrm{C} 21-\mathrm{C} 22$ & $69.4(2)$ & $\mathrm{C} 42-\mathrm{C} 43-\mathrm{C} 31-\mathrm{C} 32$ & $-178.93(18)$ \\
\hline
\end{tabular}

Table 5

Selected torsion angles $\left(^{\circ}\right)$ for (II).

\begin{tabular}{lrlr}
\hline $\mathrm{C} 2-\mathrm{N} 1-\mathrm{C} 1-\mathrm{C} 11$ & $-51.9(4)$ & $\mathrm{C} 3-\mathrm{N} 4-\mathrm{C} 41-\mathrm{C} 42$ & $-56.2(4)$ \\
$\mathrm{C} 2-\mathrm{N} 1-\mathrm{C} 1-\mathrm{C} 21$ & $-179.0(3)$ & $\mathrm{N} 4-\mathrm{C} 41-\mathrm{C} 42-\mathrm{C} 43$ & $121.6(4)$ \\
$\mathrm{N} 1-\mathrm{C} 1-\mathrm{C} 11-\mathrm{C} 12$ & $101.8(4)$ & $\mathrm{C} 41-\mathrm{C} 42-\mathrm{C} 43-\mathrm{C} 31$ & $172.9(3)$ \\
$\mathrm{N} 1-\mathrm{C} 1-\mathrm{C} 21-\mathrm{C} 22$ & $42.4(5)$ & $\mathrm{C} 42-\mathrm{C} 43-\mathrm{C} 31-\mathrm{C} 32$ & $-167.1(4)$ \\
\hline
\end{tabular}

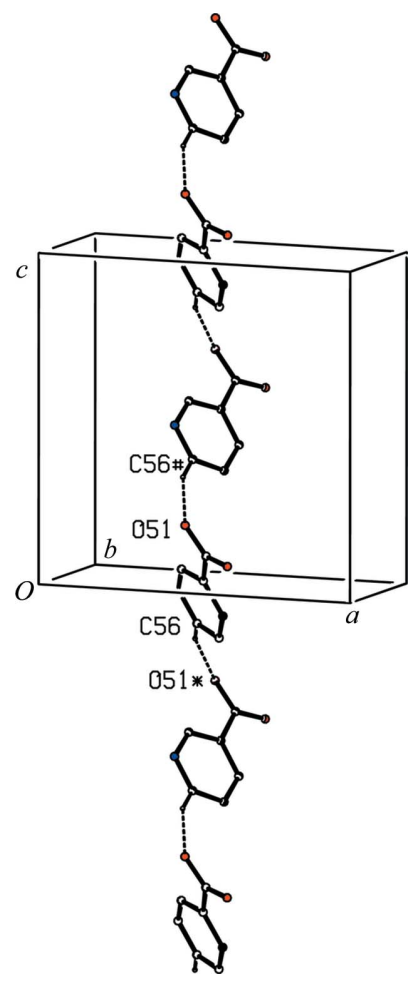

Figure 3

Part of the crystal structure of salt (I), showing the formation of a hydrogen-bonded $C(7)$ chain parallel to [001] and built from nicotinate anions only. Hydrogen bonds are shown as dashed lines and, for the sake of clarity, $\mathrm{H}$ atoms not involved in the motif shown have been omitted. Atoms marked with an asterisk or a hash symbol (\#) are at the symmetry positions $\left(x,-y+2, z-\frac{1}{2}\right)$ and $\left(x,-y+2, z+\frac{1}{2}\right)$, respectively.

(Table 2). Ion pairs of this type are linked by three independent $\mathrm{C}-\mathrm{H}$... O hydrogen bonds (Table 2) to form a threedimensional framework structure, the formation of which is
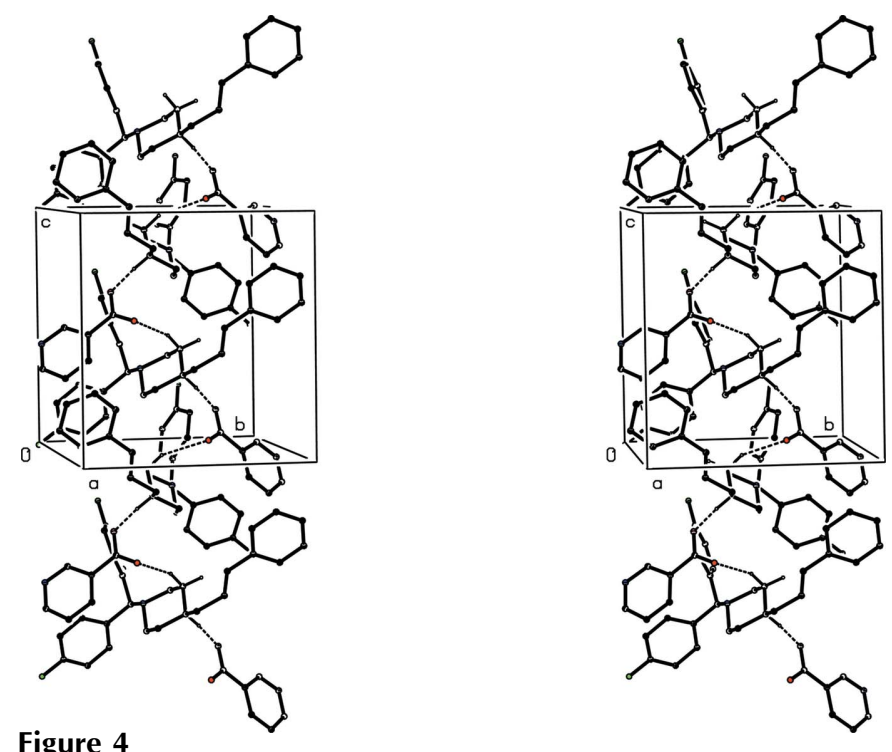

A stereoview of part of the crystal structure of salt (I), showing the formation of a hydrogen-bonded $C_{2}^{2}(7)$ chain parallel to [001] and containing alternating $\mathrm{C}-\mathrm{H} \cdots \mathrm{O}$ and $\mathrm{N}-\mathrm{H} \cdots \mathrm{O}$ hydrogen bonds, shown as dashed lines. For the sake of clarity, $\mathrm{H}$ atoms bonded to $\mathrm{C}$ atoms which are not involved in the motif shown have been omitted. 


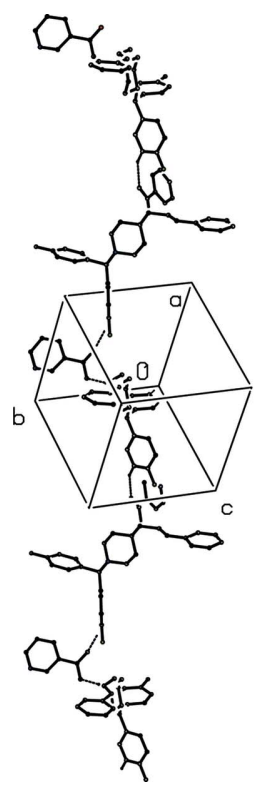

Figure 5

A stereoview of part of the crystal structure of salt (I), showing the formation of a hydrogen-bonded $C_{2}^{2}(13)$ chain parallel to [201] and containing alternating $\mathrm{C}-\mathrm{H} \cdots \mathrm{O}$ and $\mathrm{N}-\mathrm{H} \cdots \mathrm{O}$ hydrogen bonds, shown as dashed lines. For the sake of clarity, $\mathrm{H}$ atoms bonded to $\mathrm{C}$ atoms which are not involved in the motif shown have been omitted.

readily analysed in terms of three fairly simple one-dimensional substructures (Ferguson et al., 1998a,b; Gregson et al., 2000). The simplest of these substructures involves only the nicotinate component, where anions related by the $c$-glide plane at $y=1$ are linked into a $C(7)$ chain (Bernstein et al., 1995) running parallel to the [001] direction (Fig. 3). The $\mathrm{C}-$ $\mathrm{H}$... O hydrogen bond having atom $\mathrm{C} 3$ as the donor, together with the $\mathrm{N}-\mathrm{H} \cdots \mathrm{O}$ hydrogen bond, link ion pairs related by the $c$-glide plane at $y=\frac{1}{2}$ to form a $C_{2}^{2}(7)$ chain, also running parallel to the [001] direction (Fig. 4). The combination of the two chain motifs parallel to [001] generates a sheet lying parallel to (100) and such sheets are linked by the third onedimensional substructure. In this final substructure, the $\mathrm{C}-$ $\mathrm{H}$. . O hydrogen bond having atom $\mathrm{C} 13$ as the donor links ion pairs, again related by the $c$-glide plane at $y=\frac{1}{2}$, but this time forming a $C_{2}^{2}(13)$ chain running parallel to the [201] direction (Fig. 5), so completing the formation of the three-dimensional structure.

The resulting framework of (I) is modestly reinforced by two fairly long $\mathrm{C}-\mathrm{H} \cdots \pi$ (arene) hydrogen bonds (Table 2) and by an aromatic $\pi-\pi$ stacking interaction. Between the $\mathrm{C} 21-\mathrm{C} 26$ ring in the cation at $(x, y, z)$ and the C31-C36 ring in the cation at $\left(-1+x, 1-y,-\frac{1}{2}+z\right)$ there is a ring-centroid separation of 3.6986 (11) $\AA$. The dihedral angle between the ring planes is $5.02(9)^{\circ}$ and the shortest perpendicular distance from the centroid of one ring to the plane of the other is 3.3875 (8) $\AA$, corresponding to a nearly ideal ring-centroid offset of $c a 1.48 \AA$.

In (II), the five independent components are again linked into a three-dimensional framework structure by a combination of $\mathrm{O}-\mathrm{H} \cdots \mathrm{O}, \mathrm{N}-\mathrm{H} \cdots \mathrm{O}$ and $\mathrm{C}-\mathrm{H} \cdots \mathrm{O}$ hydrogen bonds
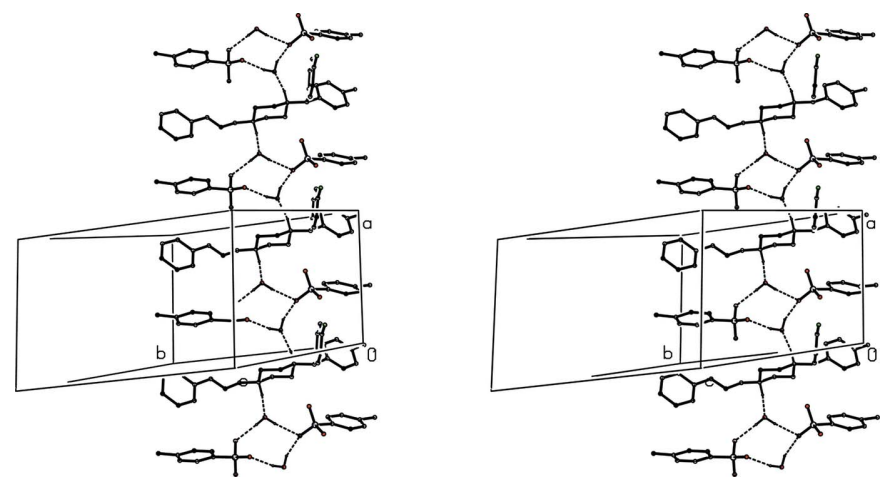

Figure 6

A stereoview of part of the crystal structure of hydrated salt (II), showing the formation of a hydrogen-bonded ribbon parallel to [100]. Hydrogen bonds are shown as dashed lines and, for the sake of clarity, $\mathrm{H}$ atoms not involved in the motif shown have been omitted and only the major orientation of the disordered anion is shown.

(Table 3), although $\mathrm{C}-\mathrm{H} \cdots \pi($ arene) hydrogen bonds and aromatic $\pi-\pi$ stacking interactions are absent from the structure. The framework of (II) is considerably more complex than that of (I) but, as for (I), its formation can readily be analysed in terms of low-dimensional substructures. The principal substructure involves only $\mathrm{O}-\mathrm{H} \cdots \mathrm{O}$ and $\mathrm{N}-$ $\mathrm{H}$... O hydrogen bonds. The five-component aggregates (Fig. 2), which are related by translation along [100], are linked to form a broad ribbon in which the cations, acting as twofold donors in $\mathrm{N}-\mathrm{H} \cdots \mathrm{O}$ hydrogen bonds, alternate with $R_{4}^{3}(10)$ rings built from the two independent anions and the two independent water molecules (Fig. 6). Four of these ribbons pass through each unit cell, with the hydrogen bonds lying approximately along the lines $(x, 0.4,0.1),(x, 0.9,0.4),(x$, $0.6,0.9)$ and $(x, 0.1,0.6)$.

The structure of (II) contains a number of short intermolecular $\mathrm{C}-\mathrm{H} \cdots \mathrm{O}$ contacts (Table 3). However, the majority of these lie either within the selected asymmetric unit or within the ribbon built from the $\mathrm{O}-\mathrm{H} \cdots \mathrm{O}$ and $\mathrm{N}-\mathrm{H} \cdots \mathrm{O}$ hydrogen bonds, or they have $\mathrm{C}-\mathrm{H} \cdots \mathrm{O}$ angles close to $140^{\circ}$, such that they cannot be regarded as structurally significant (Wood et al., 2009). Hence, the only two of these interactions which can be regarded as structurally significant hydrogen bonds influencing the overall dimensionality of the supramolecular assembly are those having atoms O51 and O61 as the acceptors, and each of these hydrogen bonds can be regarded as the basis of a simple substructure.

The $\mathrm{C}-\mathrm{H}$... O hydrogen bond involving atom $\mathrm{O} 51$ leads to the formation of a $C_{3}^{2}(16)$ chain running parallel to the [010] direction, comprising only the cation, the anion containing atom S51 and the water molecule containing atom O71, and built from components related by the $2_{1}$ screw axis along $\left(0, y, \frac{1}{4}\right)$ (Fig. 7). The effect of this chain motif is to link the [100] chains in the domain $0<z<\frac{1}{2}$ into a sheet lying parallel to (001). A second sheet of this type, related to the first by inversion, lies in the domain $\frac{1}{2}<z<1.0$. The final substructure involves, in addition to the cation, the anion containing atom S61 and the water molecule containing atom O81 (i.e. those 

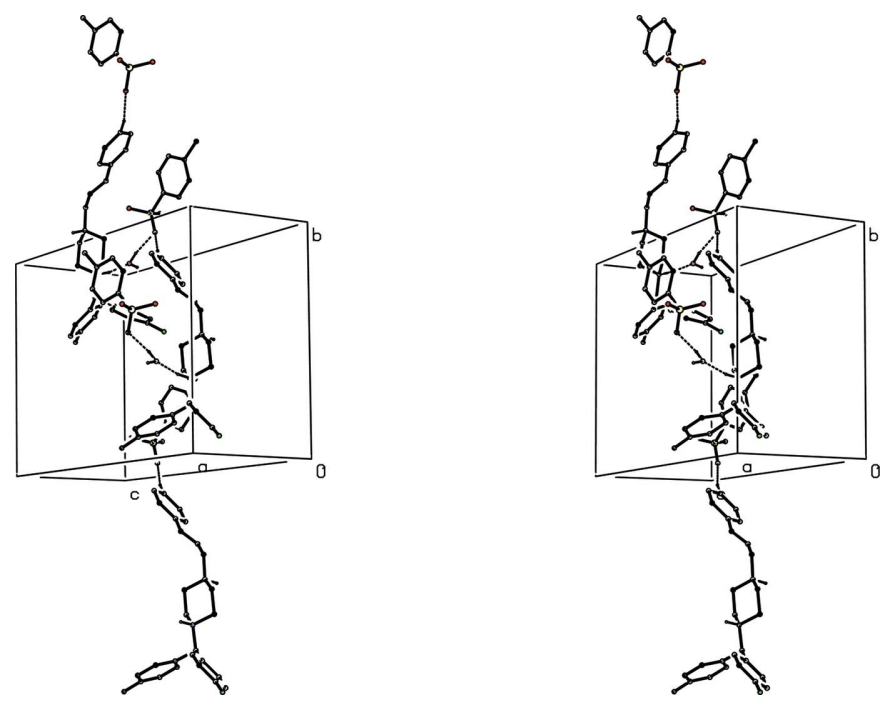

Figure 7

A stereoview of part of the crystal structure of hydrated salt (II), showing the formation of a hydrogen-bonded $C_{3}^{2}(16)$ chain parallel to [010] and incorporating the cation and only one type each of the independent anions and water molecule. Hydrogen bonds are shown as dashed lines and, for the sake of clarity, $\mathrm{H}$ atoms not involved in the motif shown have been omitted.

not participating in the chain along [010]), and it takes the form of a finite zero-dimensional motif characterized by an $R_{6}^{4}(14)$ ring (Fig. 8). The effect of this ring motif is to link directly the sheet in the domain $0<z<\frac{1}{2}$ with two adjacent sheets in the domains $\frac{1}{2}<z<1.0$ and $-\frac{1}{2}<z<0$, so completing the formation of the three-dimensional framework structure.

It is of interest briefly to compare the supramolecular assembly in some closely-related compounds with that reported here for (I) and (II). Flunarizine forms 1:1 salts with both succinic and maleic acids, and in flunarizinium hydrogen succinate, (III) (Kavitha, Yathirajan et al., 2013), the anion adopts an extended chain conformation such that the carboxylic acid - $\mathrm{OH}$ group is available as a donor in the formation of inter-anion $\mathrm{O}-\mathrm{H} \cdots \mathrm{O}$ hydrogen bonds. By contrast, the anion in flunarizinium hydrogen maleate, (IV) (Kavitha, Jasinski et al., 2013), contains a short intra-anion $\mathrm{O}-\mathrm{H} \cdots \mathrm{O}$ hydrogen bond, forming an $S(7)$ motif, and the carboxylic acid - $\mathrm{OH}$ group is not available as a donor for the formation of hydrogen bonds with other entities in the structure. In (III), a combination of $\mathrm{O}-\mathrm{H} \cdots \mathrm{O}, \mathrm{N}-\mathrm{H} \cdots \mathrm{O}$ and $\mathrm{C}-\mathrm{H} \cdots \mathrm{O}$ hydrogen bonds links the component ions into a three-dimensional framework structure. The supramolecular structure of (IV) was described in the original report as a chain, but re-examination of this structure using the published atomic coordinates shows the structure to consist of a ribbon built from alternating edge-fused $S(7)$ and $R_{3}^{3}(11)$ rings (Fig. 9). In flunarizinediium dichloride hemihydrate, (V) (Shivaprakash et al., 2014), the independent ionic components are linked by two nearly-linear charge-assisted $\mathrm{N}-\mathrm{H} \cdots \mathrm{Cl}$ hydrogen bonds and symmetry-related pairs of these ion triplets are linked by $\mathrm{O}-\mathrm{H} \cdots \mathrm{Cl}$ hydrogen bonds, where the donor is a water molecule lying across a twofold rotation axis,

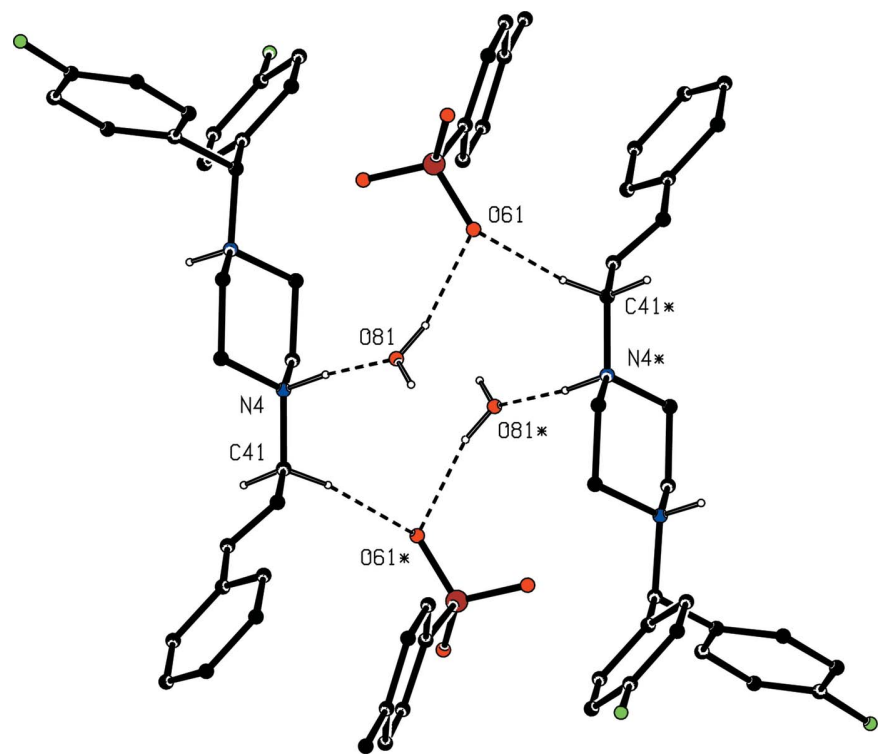

Figure 8

Part of the crystal structure of hydrated salt (II), showing the formation of a centrosymmetric hydrogen-bonded $R_{6}^{4}(14)$ motif which links adjacent (001) sheets. Hydrogen bonds are shown as dashed lines and, for the sake of clarity, components not involved in the motif shown and $\mathrm{H}$ atoms bonded to $\mathrm{C}$ atoms not involved in the motif shown have been omitted. Atoms marked with an asterisk are at the symmetry position $(-x+1$, $-y+1,-z)$.

to form a seven-component aggregate. Two independent and nearly linear $\mathrm{C}-\mathrm{H}$. C Cl hydrogen bonds link these aggregates into a complex ribbon. There are also two short $\mathrm{C}-\mathrm{H} \cdots \mathrm{O}$ contacts in the structure of $(\mathrm{V})$, but in each the $\mathrm{H} \cdots \mathrm{O}$ distance (2.67 and $2.68 \AA$ ) exceeds the sum of the van der Waals radii (2.61 А; Bondi, 1964; Rowland \& Taylor, 1996) while having $\mathrm{C}-\mathrm{H} \cdots \mathrm{O}$ angles close to $140^{\circ}$ (cf. Wood et al., 2009), so that neither of these contacts is structurally significant.
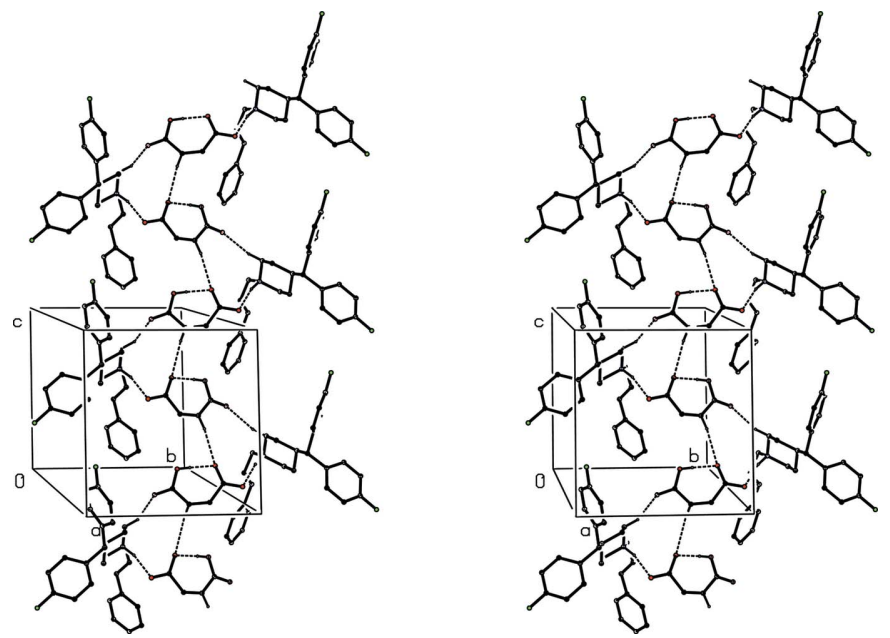

Figure 9

A stereoview of part of the crystal structure of (IV), showing the formation of a hydrogen-bonded ribbon containing alternating $S(7)$ and $R_{3}^{3}(11)$ rings. The original atomic coordinates (Kavitha, Jasinski et al., 2013) have been used. Hydrogen bonds are shown as dashed lines and, for the sake of clarity, $\mathrm{H}$ atoms not involved in the motifs shown have been omitted. 
Cinnarizine \{systematic name: 1-diphenylmethyl-4-[(2E)-3phenylprop-2-en-1-yl]piperazine\} is also a calcium-channel blocker, which differs from flunarizine only in the absence of the 4-fluoro substituents in the diarylmethyl unit. Cinnarizine forms a dihydrated 1:2 salt, (VI), with 4-toluenesulfonic acid (Kavitha, Butcher et al., 2013). Compound (VI) appears to be isomorphous with (II), although it was refined in the alternative $P 2_{1} / n$ setting, rather than in $P 2_{1} / c$ as for (II). There is very little discussion of the supramolecular assembly in the original report, apart from a listing of the $\mathrm{O}-\mathrm{H} \cdots \mathrm{O}$ and $\mathrm{N}-$ $\mathrm{H}$.. O hydrogen bonds, while $\mathrm{C}-\mathrm{H} \cdots \mathrm{O}$ hydrogen bonds were not mentioned. Re-examination of the supramolecular assembly of (VI) using the published atomic coordinates shows that it contains the same type of ribbon along [100], built from $\mathrm{O}-\mathrm{H} \cdots \mathrm{O}$ and $\mathrm{N}-\mathrm{H} \cdots \mathrm{O}$ hydrogen bonds, as found here for (II) ( $c f$. Fig. 6). In addition, there are two significant $\mathrm{C}-\mathrm{H} \cdots \mathrm{O}$ hydrogen bonds in the structure of (VI), one of which generates a $C_{3}^{2}(16)$ chain running parallel to [010], while the other generates a centrosymmetric $R_{6}^{4}(14)$ motif, entirely comparable with the action of the corresponding hydrogen bonds in (II) ( $c f$. Figs. 7 and 8). Hence (II) and (VI) are isostructural, as confirmed by a new refinement for (VI) carried out in the space group $P 2_{1} / c$ using the deposited structure-factor data. This refinement, using the coordinates of (II) as the starting point, but with the two F atoms replaced by $\mathrm{H}$ atoms, converged to $R=0.0197$ for 7891 observed reflections and 509 parameters subject to 27 restraints, with refined site occupancies for the disordered anion of 0.804 (2) and 0.196 (2), thus demonstrating clearly the isostructural nature of (II) and (VI). It is noteworthy that the absence of $\mathrm{F}$ substituents from (VI) appears to have no significant influence on the structure, apart from the minor and expected difference in the unit-cell volumes for (II) and (VI), viz. $3853.2(3) \AA^{3}$ at $200(2) \mathrm{K}$ and $3801.25(14) \AA^{3}$ at 100 (2) K, respectively.

CNK thanks the University of Mysore for research facilities and is grateful to the Principal, Maharani's Science College for
Women, Mysore, for giving permission to carry out this research.

Supporting information for this paper is available from the IUCr electronic archives (Reference: SK3553).

\section{References}

Amery, W. K. (1983). Headache, 23, 70-74.

Bernstein, J., Davis, R. E., Shimoni, L. \& Chang, N.-L. (1995). Angew. Chem. Int. Ed. Engl. 34, 1555-1573.

Boeyens, J. C. A. (1978). J. Cryst. Mol. Struct. 8, 317-320.

Bondi, A. (1964). J. Phys. Chem. 68, 441-452.

Bruker (2010). APEX2 and SAINT. Bruker AXS Inc., Madison, Wisconsin, USA.

Cremer, D. \& Pople, J. A. (1975). J. Am. Chem. Soc. 97, 1354-1358.

Fagbemi, O., Kane, K. A., McDonald, F. M., Parrett, J. R. \& Rothaul, A. L. (1984). Br. J. Pharmacol. 83, 299-304.

Ferguson, G., Glidewell, C., Gregson, R. M. \& Meehan, P. R. (1998a). Acta Cryst. B54, 129-138.

Ferguson, G., Glidewell, C., Gregson, R. M. \& Meehan, P. R. (1998b). Acta Cryst. B54, 139-150.

Flack, H. D. (1983). Acta Cryst. A39, 876-881.

Gilli, P., Bertolasi, V., Ferretti, V. \& Gilli, G. (1994). J. Am. Chem. Soc. 116, 909-915.

Gregson, R. M., Glidewell, C., Ferguson, G. \& Lough, A. J. (2000). Acta Cryst. B56, 39-57.

Holmes, B., Brogden, R. N., Heel, R. C., Speight, T. M. \& Avery, G. S. (1984). Drugs, 27, 6-44.

Hooft, R. W. W., Straver, L. H. \& Spek, A. L. (2008). J. Appl. Cryst. 41, 96-103.

Kavitha, C. N., Butcher, R. J., Jasinski, J. P., Yathirajan, H. S. \& Dayananda, A. S. (2013). Acta Cryst. E69, o485-o486.

Kavitha, C. N., Jasinski, J. P., Matar, S. M., Yathirajan, H. S. \& Ramesha, A. R. (2013). Acta Cryst. E69, o1344.

Kavitha, C. N., Yathirajan, H. S., Narayana, B., Gerber, T., van Brecht, B. \& Betz, R. (2013). Acta Cryst. E69, o260-o261.

Kavitha, C. N., Yildirim, S. Ö., Jasinski, J. P., Yathirajan, H. S. \& Butcher, R. J. (2013). Acta Cryst. E69, o142-o143.

Parsons, S., Flack, H. D. \& Wagner, T. (2013). Acta Cryst. B69, 249-259.

Rowland, R. S. \& Taylor, R. (1996). J. Phys. Chem. 100, 7384-7391.

Sheldrick, G. M. (2003). SADABS. University of Göttingen, Germany.

Sheldrick, G. M. (2008). Acta Cryst. A64, 112-122.

Sheldrick, G. M. (2014). SHELXL2014. University of Göttingen, Germany.

Shivaprakash, S., Chandrasekara Reddy, G. \& Jasinski, J. P. (2014). Acta Cryst. E70, o694-0695.

Spek, A. L. (2009). Acta Cryst. D65, 148-155.

Tarland, O. \& Flatmark, T. (1999). Neuropharmacology, 38, 879-882.

Wood, P. A., Allen, F. H. \& Pidcock, E. (2009). CrystEngComm, 11, 15631571. 


\section{supporting information}

Acta Cryst. (2014). C70, 805-811 [doi:10.1107/S2053229614016532]

\section{Three-dimensional hydrogen-bonded framework structures in flunarizinium nicotinate and flunarizinediium bis(4-toluenesulfonate) dihydrate}

\section{Channappa N. Kavitha, Hemmige S. Yathirajan, Manpreet Kaur, Eric C. Hosten, Richard Betz and Christopher Glidewell}

\section{Computing details}

For both compounds, data collection: APEX2 (Bruker, 2010); cell refinement: SAINT (Bruker, 2010); data reduction: SAINT (Bruker, 2010); program(s) used to solve structure: SHELXS97 (Sheldrick, 2008); program(s) used to refine structure: SHELXL2014 (Sheldrick, 2014); molecular graphics: PLATON (Spek, 2009); software used to prepare material for publication: SHELXL2014 (Sheldrick, 2014) and PLATON (Spek, 2009).

(I) 4-Bis[(4-fluorophenyl)methyl]-1-[(2E)-3-phenylprop-2-en-1-yl]piperazin-1-ium pyridine-3-carboxylate

Crystal data

$\mathrm{C}_{26} \mathrm{H}_{27} \mathrm{~F}_{2} \mathrm{~N}_{2} \cdot \mathrm{C}_{6} \mathrm{H}_{4} \mathrm{NO}_{2}$

$M_{r}=527.60$

Monoclinic, $P c$

$a=10.8536(4) \AA$

$b=10.8103(4) \AA$

$c=11.3901(4) \AA$

$\beta=92.717(2)^{\circ}$

$V=1334.91(8) \AA^{3}$

$Z=2$

\section{Data collection}

Bruker APEXII CCD area-detector diffractometer

$\varphi$ and $\omega$ scans

Absorption correction: multi-scan

(SADABS; Sheldrick, 2003)

$T_{\text {min }}=0.873, T_{\max }=0.988$

23278 measured reflections

Refinement

Refinement on $F^{2}$

Least-squares matrix: full

$R\left[F^{2}>2 \sigma\left(F^{2}\right)\right]=0.030$

$w R\left(F^{2}\right)=0.081$

$S=1.03$

6092 reflections

352 parameters

2 restraints
$F(000)=556$

$D_{\mathrm{x}}=1.313 \mathrm{Mg} \mathrm{m}^{-3}$

Mo $K \alpha$ radiation, $\lambda=0.71073 \AA$

Cell parameters from 6092 reflections

$\theta=1.9-28.4^{\circ}$

$\mu=0.09 \mathrm{~mm}^{-1}$

$T=200 \mathrm{~K}$

Plate, colourless

$0.54 \times 0.49 \times 0.13 \mathrm{~mm}$

6092 independent reflections

5770 reflections with $I>2 \sigma(I)$

$R_{\text {int }}=0.027$

$\theta_{\text {max }}=28.4^{\circ}, \theta_{\text {min }}=1.9^{\circ}$

$h=-14 \rightarrow 14$

$k=-14 \rightarrow 14$

$l=-15 \rightarrow 14$

Hydrogen site location: mixed

$\mathrm{H}$-atom parameters constrained

$w=1 /\left[\sigma^{2}\left(F_{\mathrm{o}}^{2}\right)+(0.045 P)^{2}+0.1632 P\right]$

where $P=\left(F_{\mathrm{o}}^{2}+2 F_{\mathrm{c}}^{2}\right) / 3$

$(\Delta / \sigma)_{\max }<0.001$

$\Delta \rho_{\max }=0.15 \mathrm{e}^{-3}$

$\Delta \rho_{\min }=-0.18$ e $\AA^{-3}$ 
Absolute structure: Flack $x$ parameter (Flack, 1983) determined using 2539 quotients $[(\mathrm{I}+)-(\mathrm{I}-)] /[(\mathrm{I}+)+(\mathrm{I}-)]($ Parsons et al., 2013$)$

Absolute structure parameter: 0.10 (15)

Special details

Geometry. All e.s.d.'s (except the e.s.d. in the dihedral angle between two 1.s. planes) are estimated using the full covariance matrix. The cell e.s.d.'s are taken into account individually in the estimation of e.s.d.'s in distances, angles and torsion angles; correlations between e.s.d.'s in cell parameters are only used when they are defined by crystal symmetry. An approximate (isotropic) treatment of cell e.s.d.'s is used for estimating e.s.d.'s involving 1.s. planes.

Fractional atomic coordinates and isotropic or equivalent isotropic displacement parameters $\left(\AA^{2}\right)$

\begin{tabular}{|c|c|c|c|c|}
\hline & $x$ & $y$ & $z$ & $U_{\text {iso }} * / U_{\text {eq }}$ \\
\hline N1 & $0.17850(12)$ & $0.43015(13)$ & $0.31935(12)$ & $0.0250(3)$ \\
\hline $\mathrm{C} 2$ & $0.19249(15)$ & $0.55279(15)$ & $0.37393(15)$ & $0.0261(3)$ \\
\hline $\mathrm{H} 2 \mathrm{~A}$ & 0.1416 & 0.5572 & 0.4437 & $0.031^{*}$ \\
\hline H2B & 0.1625 & 0.6169 & 0.3175 & $0.031^{*}$ \\
\hline $\mathrm{C} 3$ & $0.32579(15)$ & $0.57830(16)$ & $0.41056(14)$ & $0.0268(3)$ \\
\hline $\mathrm{H} 3 \mathrm{~A}$ & 0.3330 & 0.6617 & 0.4462 & $0.032^{*}$ \\
\hline H3B & 0.3547 & 0.5170 & 0.4703 & $0.032^{*}$ \\
\hline N4 & $0.40422(12)$ & $0.57106(13)$ & $0.30680(12)$ & $0.0244(3)$ \\
\hline $\mathrm{H} 4$ & 0.3770 & 0.6362 & 0.2515 & $0.029^{*}$ \\
\hline $\mathrm{C} 5$ & $0.38561(15)$ & $0.44918(16)$ & $0.24771(15)$ & $0.0276(3)$ \\
\hline $\mathrm{H} 5 \mathrm{~A}$ & 0.4157 & 0.3824 & 0.3012 & $0.033^{*}$ \\
\hline H5B & 0.4341 & 0.4463 & 0.1763 & $0.033^{*}$ \\
\hline C6 & $0.25047(15)$ & $0.42811(16)$ & $0.21400(15)$ & $0.0273(3)$ \\
\hline H6A & 0.2206 & 0.4936 & 0.1589 & $0.033^{*}$ \\
\hline H6B & 0.2400 & 0.3472 & 0.1740 & $0.033^{*}$ \\
\hline $\mathrm{C} 1$ & $0.04617(14)$ & $0.40285(15)$ & $0.29532(15)$ & $0.0250(3)$ \\
\hline H1 & 0.0071 & 0.4774 & 0.2573 & $0.030 *$ \\
\hline C11 & $-0.01644(14)$ & $0.37958(15)$ & $0.41020(15)$ & $0.0251(3)$ \\
\hline $\mathrm{C} 12$ & $-0.14102(15)$ & $0.40862(16)$ & $0.41860(16)$ & $0.0302(4)$ \\
\hline H12 & -0.1844 & 0.4472 & 0.3541 & $0.036^{*}$ \\
\hline $\mathrm{C} 13$ & $-0.20273(17)$ & $0.38200(18)$ & $0.51973(18)$ & $0.0358(4)$ \\
\hline H13 & -0.2879 & 0.4003 & 0.5247 & $0.043^{*}$ \\
\hline $\mathrm{C} 14$ & $-0.13720(18)$ & $0.32879(16)$ & $0.61156(17)$ & $0.0345(4)$ \\
\hline F14 & $-0.19601(13)$ & $0.30702(13)$ & $0.71334(11)$ & $0.0516(3)$ \\
\hline $\mathrm{C} 15$ & $-0.01478(18)$ & $0.29758(17)$ & $0.60796(17)$ & $0.0336(4)$ \\
\hline H15 & 0.0275 & 0.2594 & 0.6733 & $0.040^{*}$ \\
\hline $\mathrm{C} 16$ & $0.04540(16)$ & $0.32364(16)$ & $0.50559(16)$ & $0.0294(3)$ \\
\hline H16 & 0.1301 & 0.3028 & 0.5009 & $0.035^{*}$ \\
\hline $\mathrm{C} 21$ & $0.02675(15)$ & $0.29468(16)$ & $0.21188(14)$ & $0.0265(3)$ \\
\hline $\mathrm{C} 22$ & $0.05734(17)$ & $0.17386(17)$ & $0.24553(18)$ & $0.0346(4)$ \\
\hline $\mathrm{H} 22$ & 0.0922 & 0.1585 & 0.3222 & $0.041^{*}$ \\
\hline $\mathrm{C} 23$ & $0.0372(2)$ & 0.07615 (19) & $0.1681(2)$ & $0.0435(5)$ \\
\hline $\mathrm{H} 23$ & 0.0577 & -0.0061 & 0.1911 & $0.052^{*}$ \\
\hline $\mathrm{C} 24$ & $-0.01274(19)$ & $0.1004(2)$ & $0.0577(2)$ & $0.0454(5)$ \\
\hline F24 & $-0.03385(15)$ & $0.00390(16)$ & $-0.01654(15)$ & $0.0702(5)$ \\
\hline
\end{tabular}




\begin{tabular}{|c|c|c|c|c|}
\hline $\mathrm{C} 25$ & $-0.04382(18)$ & $0.2171(2)$ & $0.02057(18)$ & $0.0437(5)$ \\
\hline $\mathrm{H} 25$ & -0.0782 & 0.2311 & -0.0565 & $0.052 *$ \\
\hline $\mathrm{C} 26$ & $-0.02378(17)$ & $0.31482(19)$ & $0.09868(16)$ & $0.0334(4)$ \\
\hline $\mathrm{H} 26$ & -0.0449 & 0.3965 & 0.0745 & $0.040^{*}$ \\
\hline $\mathrm{C} 41$ & $0.53859(16)$ & $0.58793(18)$ & $0.34023(17)$ & $0.0321(4)$ \\
\hline H41A & 0.5671 & 0.5174 & 0.3899 & $0.039^{*}$ \\
\hline H41B & 0.5860 & 0.5870 & 0.2681 & $0.039^{*}$ \\
\hline $\mathrm{C} 42$ & $0.56485(16)$ & $0.70599(18)$ & $0.40543(17)$ & $0.0327(4)$ \\
\hline $\mathrm{H} 42$ & 0.5461 & 0.7823 & 0.3673 & $0.039^{*}$ \\
\hline $\mathrm{C} 43$ & $0.61323(16)$ & $0.70778(17)$ & $0.51436(16)$ & $0.0313(4)$ \\
\hline H43 & 0.6269 & 0.6293 & 0.5502 & $0.038 *$ \\
\hline C31 & $0.64836(15)$ & $0.81561(17)$ & $0.58665(15)$ & $0.0299(4)$ \\
\hline $\mathrm{C} 32$ & $0.70012(17)$ & $0.79671(19)$ & $0.69949(17)$ & $0.0342(4)$ \\
\hline H32 & 0.7088 & 0.7147 & 0.7287 & $0.041^{*}$ \\
\hline $\mathrm{C} 33$ & $0.73911(18)$ & $0.8947(2)$ & $0.77006(18)$ & $0.0401(4)$ \\
\hline $\mathrm{H} 33$ & 0.7747 & 0.8797 & 0.8466 & $0.048^{*}$ \\
\hline $\mathrm{C} 34$ & $0.72614(19)$ & $1.0147(2)$ & $0.7288(2)$ & $0.0425(4)$ \\
\hline H34 & 0.7532 & 1.0824 & 0.7767 & $0.051^{*}$ \\
\hline C35 & $0.67351(19)$ & $1.03563(19)$ & $0.6173(2)$ & $0.0417(4)$ \\
\hline H35 & 0.6637 & 1.1180 & 0.5893 & $0.050 *$ \\
\hline $\mathrm{C} 36$ & $0.63521(18)$ & $0.93764(18)$ & $0.54642(18)$ & $0.0354(4)$ \\
\hline H36 & 0.5998 & 0.9532 & 0.4700 & $0.042 *$ \\
\hline N51 & $0.26141(19)$ & $1.03197(17)$ & $-0.06212(17)$ & $0.0492(5)$ \\
\hline C52 & $0.28936(19)$ & $0.93447(18)$ & $0.00654(17)$ & $0.0370(4)$ \\
\hline H52 & 0.2473 & 0.9261 & 0.0774 & $0.044 *$ \\
\hline $\mathrm{C} 53$ & $0.37557(16)$ & $0.84493(16)$ & $-0.01882(15)$ & $0.0290(3)$ \\
\hline C54 & $0.43615(18)$ & $0.85725(19)$ & $-0.12234(17)$ & $0.0378(4)$ \\
\hline H54 & 0.4972 & 0.7991 & -0.1426 & $0.045^{*}$ \\
\hline C55 & $0.4063(2)$ & $0.9557(2)$ & $-0.19576(19)$ & $0.0464(5)$ \\
\hline H55 & 0.4447 & 0.9650 & -0.2685 & $0.056^{*}$ \\
\hline C56 & $0.3202(2)$ & $1.0397(2)$ & $-0.1618(2)$ & $0.0480(5)$ \\
\hline H56 & 0.3015 & 1.1076 & -0.2125 & $0.058^{*}$ \\
\hline C57 & $0.40362(16)$ & $0.73877(16)$ & $0.06383(15)$ & $0.0278(3)$ \\
\hline O51 & $0.33722(13)$ & $0.73420(12)$ & $0.15347(11)$ & $0.0360(3)$ \\
\hline O52 & $0.48540(13)$ & $0.66376(13)$ & $0.04174(13)$ & $0.0407(3)$ \\
\hline
\end{tabular}

Atomic displacement parameters $\left(\AA^{2}\right)$

\begin{tabular}{lllllll}
\hline & $U^{11}$ & $U^{22}$ & $U^{33}$ & $U^{12}$ & $U^{13}$ & $U^{23}$ \\
\hline $\mathrm{N} 1$ & $0.0236(6)$ & $0.0273(7)$ & $0.0243(7)$ & $-0.0025(5)$ & $0.0046(5)$ & $-0.0024(5)$ \\
$\mathrm{C} 2$ & $0.0252(7)$ & $0.0279(8)$ & $0.0255(8)$ & $-0.0009(6)$ & $0.0040(6)$ & $-0.0033(6)$ \\
$\mathrm{C} 3$ & $0.0277(8)$ & $0.0318(8)$ & $0.0212(8)$ & $-0.0038(6)$ & $0.0037(6)$ & $-0.0027(6)$ \\
$\mathrm{N} 4$ & $0.0222(6)$ & $0.0288(7)$ & $0.0222(7)$ & $-0.0022(5)$ & $0.0019(5)$ & $0.0008(5)$ \\
$\mathrm{C} 5$ & $0.0259(8)$ & $0.0296(8)$ & $0.0277(8)$ & $-0.0003(6)$ & $0.0053(6)$ & $-0.0025(7)$ \\
$\mathrm{C} 6$ & $0.0262(8)$ & $0.0316(8)$ & $0.0245(8)$ & $-0.0026(6)$ & $0.0046(6)$ & $-0.0039(6)$ \\
$\mathrm{C} 1$ & $0.0230(7)$ & $0.0257(7)$ & $0.0262(8)$ & $-0.0001(6)$ & $0.0008(6)$ & $0.0006(6)$ \\
$\mathrm{C} 11$ & $0.0247(7)$ & $0.0235(7)$ & $0.0274(8)$ & $-0.0033(6)$ & $0.0028(6)$ & $-0.0037(6)$ \\
$\mathrm{C} 12$ & $0.0245(7)$ & $0.0332(8)$ & $0.0327(9)$ & $-0.0001(6)$ & $0.0000(6)$ & $-0.0040(7)$
\end{tabular}




\begin{tabular}{|c|c|c|c|c|c|c|}
\hline $\mathrm{C} 13$ & $0.0271(8)$ & $0.0378(9)$ & $0.0432(10)$ & $-0.0044(7)$ & $0.0089(7)$ & $-0.0084(8)$ \\
\hline $\mathrm{C} 14$ & $0.0405(9)$ & $0.0319(8)$ & $0.0323(9)$ & $-0.0098(7)$ & $0.0141(7)$ & $-0.0047(7)$ \\
\hline F14 & $0.0582(8)$ & $0.0568(8)$ & $0.0419(7)$ & $-0.0109(6)$ & $0.0256(6)$ & $-0.0005(6)$ \\
\hline $\mathrm{C} 15$ & $0.0407(9)$ & $0.0307(8)$ & $0.0294(9)$ & $-0.0036(7)$ & $0.0023(7)$ & $0.0016(7)$ \\
\hline $\mathrm{C} 16$ & $0.0266(8)$ & $0.0298(8)$ & $0.0319(9)$ & $0.0001(6)$ & $0.0027(6)$ & $0.0002(7)$ \\
\hline $\mathrm{C} 21$ & $0.0221(7)$ & $0.0305(8)$ & $0.0270(8)$ & $-0.0039(6)$ & $0.0020(6)$ & $-0.0027(7)$ \\
\hline $\mathrm{C} 22$ & $0.0351(9)$ & $0.0322(9)$ & $0.0364(10)$ & $-0.0002(7)$ & $0.0018(7)$ & $-0.0027(7)$ \\
\hline $\mathrm{C} 23$ & $0.0410(10)$ & $0.0352(10)$ & $0.0550(13)$ & $-0.0023(8)$ & $0.0095(9)$ & $-0.0120(9)$ \\
\hline $\mathrm{C} 24$ & $0.0364(10)$ & $0.0527(12)$ & $0.0479(12)$ & $-0.0120(9)$ & $0.0108(9)$ & $-0.0264(10)$ \\
\hline F24 & $0.0647(9)$ & $0.0721(10)$ & $0.0744(10)$ & $-0.0163(8)$ & $0.0103(8)$ & $-0.0485(8)$ \\
\hline $\mathrm{C} 25$ & $0.0344(9)$ & $0.0675(14)$ & $0.0292(9)$ & $-0.0095(9)$ & $0.0023(8)$ & $-0.0119(9)$ \\
\hline $\mathrm{C} 26$ & $0.0289(8)$ & $0.0439(10)$ & $0.0278(9)$ & $-0.0041(7)$ & $0.0029(7)$ & -0.0008 \\
\hline $\mathrm{C} 41$ & $0.0227(7)$ & $0.0409(9)$ & $0.0327(9)$ & $-0.0019(7)$ & $0.0003(6)$ & $0.0000(7)$ \\
\hline $\mathrm{C} 42$ & $0.0262(8)$ & $0.0358(9)$ & $0.0359(10)$ & $-0.0046(7)$ & $0.0004(7)$ & $0.0046(8)$ \\
\hline $\mathrm{C} 43$ & $0.0276(8)$ & $0.0323(8)$ & $0.0341(9)$ & $0.0022(7)$ & $0.0022(7)$ & $0.0020(7)$ \\
\hline C31 & $0.0226(7)$ & $0.0364(9)$ & $0.0309(9)$ & $0.0012(6)$ & $0.0026(6)$ & $0.0005(7)$ \\
\hline $\mathrm{C} 32$ & $0.0300(8)$ & $0.0392(9)$ & $0.0334(9)$ & $0.0048(7)$ & $0.0013(7)$ & $0.0025(8)$ \\
\hline $\mathrm{C} 33$ & $0.0338(9)$ & $0.0545(12)$ & $0.0316(9)$ & $0.0006(8)$ & $-0.0019(7)$ & $-0.0019(9)$ \\
\hline $\mathrm{C} 34$ & $0.0359(9)$ & $0.0458(11)$ & $0.0458(11)$ & $-0.0066(8)$ & $0.0020(8)$ & $-0.0105(9)$ \\
\hline $\mathrm{C} 35$ & $0.0393(10)$ & $0.0342(9)$ & $0.0516(12)$ & $-0.0031(8)$ & $0.0031(9)$ & $0.0008(9)$ \\
\hline $\mathrm{C} 36$ & $0.0331(9)$ & $0.0392(9)$ & $0.0335(9)$ & $0.0003(7)$ & $-0.0012(7)$ & $0.0046(8)$ \\
\hline N51 & $0.0569(11)$ & $0.0434(10)$ & $0.0469(11)$ & $0.0060(9)$ & $-0.0031(9)$ & $0.0113(8)$ \\
\hline $\mathrm{C} 52$ & $0.0416(10)$ & $0.0388(10)$ & $0.0309(9)$ & $0.0023(8)$ & $0.0040(7)$ & $0.0052(8)$ \\
\hline $\mathrm{C} 53$ & $0.0299(8)$ & $0.0299(8)$ & $0.0271(8)$ & $-0.0083(6)$ & $-0.0002(6)$ & $-0.0008(7)$ \\
\hline $\mathrm{C} 54$ & $0.0387(9)$ & $0.0413(10)$ & $0.0342(10)$ & $-0.0121(8)$ & $0.0091(8)$ & $-0.0037(8)$ \\
\hline $\mathrm{C} 55$ & $0.0548(12)$ & $0.0545(12)$ & $0.0302(10)$ & $-0.0224(10)$ & $0.0054(9)$ & $0.0076(9)$ \\
\hline $\mathrm{C} 56$ & $0.0563(13)$ & $0.0447(11)$ & $0.0418(12)$ & $-0.0128(10)$ & $-0.0097(10)$ & $0.0161(9)$ \\
\hline $\mathrm{C} 57$ & $0.0280(7)$ & $0.0275(8)$ & $0.0279(8)$ & $-0.0049(6)$ & $-0.0006(6)$ & $-0.0020(6)$ \\
\hline O51 & $0.0428(7)$ & $0.0361(6)$ & $0.0298(6)$ & $0.0054(6)$ & $0.0086(5)$ & $0.0067(5)$ \\
\hline O52 & $0.0382(7)$ & $0.0389(7)$ & $0.0457(8)$ & $0.0061(6)$ & $0.0082(6)$ & $-0.0006(6)$ \\
\hline
\end{tabular}

Geometric parameters $\left(\AA,{ }^{\circ}\right)$

\begin{tabular}{llll}
\hline $\mathrm{N} 1-\mathrm{C} 6$ & $1.463(2)$ & $\mathrm{C} 24-\mathrm{F} 24$ & $1.355(2)$ \\
$\mathrm{N} 1-\mathrm{C} 2$ & $1.469(2)$ & $\mathrm{C} 24-\mathrm{C} 25$ & $1.368(3)$ \\
$\mathrm{N} 1-\mathrm{C} 1$ & $1.479(2)$ & $\mathrm{C} 25-\mathrm{C} 26$ & $1.391(3)$ \\
$\mathrm{C} 2-\mathrm{C} 3$ & $1.512(2)$ & $\mathrm{C} 25-\mathrm{H} 25$ & 0.9500 \\
$\mathrm{C} 2-\mathrm{H} 2 \mathrm{~A}$ & 0.9900 & $\mathrm{C} 26-\mathrm{H} 26$ & 0.9500 \\
$\mathrm{C} 2-\mathrm{H} 2 \mathrm{~B}$ & 0.9900 & $\mathrm{C} 41-\mathrm{C} 42$ & $1.497(3)$ \\
$\mathrm{C} 3-\mathrm{N} 4$ & $1.491(2)$ & $\mathrm{C} 41-\mathrm{H} 41 \mathrm{~A}$ & 0.9900 \\
$\mathrm{C} 3-\mathrm{H} 3 \mathrm{~A}$ & 0.9900 & $\mathrm{C} 41-\mathrm{H} 41 \mathrm{~B}$ & 0.9900 \\
$\mathrm{C} 3-\mathrm{H} 3 \mathrm{~B}$ & 0.9900 & $\mathrm{C} 42-\mathrm{C} 43$ & $1.324(3)$ \\
$\mathrm{N} 4-\mathrm{C} 5$ & $1.489(2)$ & $\mathrm{C} 42-\mathrm{H} 42$ & 0.9500 \\
$\mathrm{~N} 4-\mathrm{C} 41$ & $1.501(2)$ & $\mathrm{C} 43-\mathrm{C} 31$ & $1.467(3)$ \\
$\mathrm{N} 4-\mathrm{H} 4$ & 0.9807 & $\mathrm{C} 43-\mathrm{H} 43$ & 0.9500 \\
$\mathrm{C} 5-\mathrm{C} 6$ & $1.516(2)$ & $\mathrm{C} 31-\mathrm{C} 32$ & $1.393(3)$ \\
$\mathrm{C} 5-\mathrm{H} 5 \mathrm{~A}$ & 0.9900 & $\mathrm{C} 31-\mathrm{C} 36$ & $1.402(3)$ \\
$\mathrm{C} 5-\mathrm{H} 5 \mathrm{~B}$ & 0.9900 & $\mathrm{C} 32-\mathrm{C} 33$ & $1.384(3)$
\end{tabular}




\begin{tabular}{|c|c|c|c|}
\hline C6-H6A & 0.9900 & $\mathrm{C} 32-\mathrm{H} 32$ & 0.9500 \\
\hline C6-H6B & 0.9900 & $\mathrm{C} 33-\mathrm{C} 34$ & $1.385(3)$ \\
\hline $\mathrm{C} 1-\mathrm{C} 21$ & $1.515(2)$ & $\mathrm{C} 33-\mathrm{H} 33$ & 0.9500 \\
\hline $\mathrm{C} 1-\mathrm{C} 11$ & $1.524(2)$ & $\mathrm{C} 34-\mathrm{C} 35$ & $1.386(3)$ \\
\hline $\mathrm{C} 1-\mathrm{H} 1$ & 1.0000 & C34-H34 & 0.9500 \\
\hline $\mathrm{C} 11-\mathrm{C} 16$ & $1.388(2)$ & $\mathrm{C} 35-\mathrm{C} 36$ & $1.384(3)$ \\
\hline $\mathrm{C} 11-\mathrm{C} 12$ & $1.396(2)$ & C35-H35 & 0.9500 \\
\hline $\mathrm{C} 12-\mathrm{C} 13$ & $1.390(3)$ & $\mathrm{C} 36-\mathrm{H} 36$ & 0.9500 \\
\hline $\mathrm{C} 12-\mathrm{H} 12$ & 0.9500 & N51-C56 & $1.332(3)$ \\
\hline $\mathrm{C} 13-\mathrm{C} 14$ & $1.364(3)$ & N51-C52 & $1.339(3)$ \\
\hline C13-H13 & 0.9500 & $\mathrm{C} 52-\mathrm{C} 53$ & $1.386(3)$ \\
\hline $\mathrm{C} 14-\mathrm{F} 14$ & $1.370(2)$ & $\mathrm{C} 52-\mathrm{H} 52$ & 0.9500 \\
\hline $\mathrm{C} 14-\mathrm{C} 15$ & $1.373(3)$ & $\mathrm{C} 53-\mathrm{C} 54$ & $1.384(2)$ \\
\hline $\mathrm{C} 15-\mathrm{C} 16$ & $1.392(3)$ & $\mathrm{C} 53-\mathrm{C} 57$ & $1.506(2)$ \\
\hline C15-H15 & 0.9500 & $\mathrm{C} 54-\mathrm{C} 55$ & $1.383(3)$ \\
\hline $\mathrm{C} 16-\mathrm{H} 16$ & 0.9500 & C54-H54 & 0.9500 \\
\hline $\mathrm{C} 21-\mathrm{C} 26$ & $1.394(2)$ & $\mathrm{C} 55-\mathrm{C} 56$ & $1.371(4)$ \\
\hline $\mathrm{C} 21-\mathrm{C} 22$ & $1.397(3)$ & $\mathrm{C} 55-\mathrm{H} 55$ & 0.9500 \\
\hline $\mathrm{C} 22-\mathrm{C} 23$ & $1.387(3)$ & $\mathrm{C} 56-\mathrm{H} 56$ & 0.9500 \\
\hline $\mathrm{C} 22-\mathrm{H} 22$ & 0.9500 & $\mathrm{C} 57-\mathrm{O} 52$ & $1.237(2)$ \\
\hline $\mathrm{C} 23-\mathrm{C} 24$ & $1.371(4)$ & $\mathrm{C} 57-\mathrm{O} 51$ & $1.278(2)$ \\
\hline $\mathrm{C} 23-\mathrm{H} 23$ & 0.9500 & & \\
\hline $\mathrm{C} 6-\mathrm{N} 1-\mathrm{C} 2$ & $108.19(12)$ & $\mathrm{C} 21-\mathrm{C} 22-\mathrm{H} 22$ & 119.7 \\
\hline $\mathrm{C} 6-\mathrm{N} 1-\mathrm{C} 1$ & $113.38(12)$ & $\mathrm{C} 24-\mathrm{C} 23-\mathrm{C} 22$ & $118.8(2)$ \\
\hline $\mathrm{C} 2-\mathrm{N} 1-\mathrm{C} 1$ & $109.77(12)$ & $\mathrm{C} 24-\mathrm{C} 23-\mathrm{H} 23$ & 120.6 \\
\hline $\mathrm{N} 1-\mathrm{C} 2-\mathrm{C} 3$ & $111.08(13)$ & $\mathrm{C} 22-\mathrm{C} 23-\mathrm{H} 23$ & 120.6 \\
\hline $\mathrm{N} 1-\mathrm{C} 2-\mathrm{H} 2 \mathrm{~A}$ & 109.4 & $\mathrm{~F} 24-\mathrm{C} 24-\mathrm{C} 25$ & $119.0(2)$ \\
\hline $\mathrm{C} 3-\mathrm{C} 2-\mathrm{H} 2 \mathrm{~A}$ & 109.4 & $\mathrm{~F} 24-\mathrm{C} 24-\mathrm{C} 23$ & $118.2(2)$ \\
\hline $\mathrm{N} 1-\mathrm{C} 2-\mathrm{H} 2 \mathrm{~B}$ & 109.4 & $\mathrm{C} 25-\mathrm{C} 24-\mathrm{C} 23$ & $122.72(18)$ \\
\hline $\mathrm{C} 3-\mathrm{C} 2-\mathrm{H} 2 \mathrm{~B}$ & 109.4 & $\mathrm{C} 24-\mathrm{C} 25-\mathrm{C} 26$ & $118.30(19)$ \\
\hline $\mathrm{H} 2 \mathrm{~A}-\mathrm{C} 2-\mathrm{H} 2 \mathrm{~B}$ & 108.0 & $\mathrm{C} 24-\mathrm{C} 25-\mathrm{H} 25$ & 120.8 \\
\hline $\mathrm{N} 4-\mathrm{C} 3-\mathrm{C} 2$ & $110.27(13)$ & $\mathrm{C} 26-\mathrm{C} 25-\mathrm{H} 25$ & 120.8 \\
\hline $\mathrm{N} 4-\mathrm{C} 3-\mathrm{H} 3 \mathrm{~A}$ & 109.6 & $\mathrm{C} 25-\mathrm{C} 26-\mathrm{C} 21$ & $121.03(19)$ \\
\hline $\mathrm{C} 2-\mathrm{C} 3-\mathrm{H} 3 \mathrm{~A}$ & 109.6 & $\mathrm{C} 25-\mathrm{C} 26-\mathrm{H} 26$ & 119.5 \\
\hline $\mathrm{N} 4-\mathrm{C} 3-\mathrm{H} 3 \mathrm{~B}$ & 109.6 & $\mathrm{C} 21-\mathrm{C} 26-\mathrm{H} 26$ & 119.5 \\
\hline $\mathrm{C} 2-\mathrm{C} 3-\mathrm{H} 3 \mathrm{~B}$ & 109.6 & $\mathrm{C} 42-\mathrm{C} 41-\mathrm{N} 4$ & $112.91(15)$ \\
\hline $\mathrm{H} 3 \mathrm{~A}-\mathrm{C} 3-\mathrm{H} 3 \mathrm{~B}$ & 108.1 & $\mathrm{C} 42-\mathrm{C} 41-\mathrm{H} 41 \mathrm{~A}$ & 109.0 \\
\hline $\mathrm{C} 5-\mathrm{N} 4-\mathrm{C} 3$ & $109.54(12)$ & $\mathrm{N} 4-\mathrm{C} 41-\mathrm{H} 41 \mathrm{~A}$ & 109.0 \\
\hline $\mathrm{C} 5-\mathrm{N} 4-\mathrm{C} 41$ & $109.36(13)$ & $\mathrm{C} 42-\mathrm{C} 41-\mathrm{H} 41 \mathrm{~B}$ & 109.0 \\
\hline $\mathrm{C} 3-\mathrm{N} 4-\mathrm{C} 41$ & $112.14(13)$ & $\mathrm{N} 4-\mathrm{C} 41-\mathrm{H} 41 \mathrm{~B}$ & 109.0 \\
\hline $\mathrm{C} 5-\mathrm{N} 4-\mathrm{H} 4$ & 108.3 & $\mathrm{H} 41 \mathrm{~A}-\mathrm{C} 41-\mathrm{H} 41 \mathrm{~B}$ & 107.8 \\
\hline $\mathrm{C} 3-\mathrm{N} 4-\mathrm{H} 4$ & 107.8 & $\mathrm{C} 43-\mathrm{C} 42-\mathrm{C} 41$ & $122.33(17)$ \\
\hline $\mathrm{C} 41-\mathrm{N} 4-\mathrm{H} 4$ & 109.6 & $\mathrm{C} 43-\mathrm{C} 42-\mathrm{H} 42$ & 118.8 \\
\hline $\mathrm{N} 4-\mathrm{C} 5-\mathrm{C} 6$ & $110.88(13)$ & $\mathrm{C} 41-\mathrm{C} 42-\mathrm{H} 42$ & 118.8 \\
\hline $\mathrm{N} 4-\mathrm{C} 5-\mathrm{H} 5 \mathrm{~A}$ & 109.5 & $\mathrm{C} 42-\mathrm{C} 43-\mathrm{C} 31$ & $128.20(17)$ \\
\hline $\mathrm{C} 6-\mathrm{C} 5-\mathrm{H} 5 \mathrm{~A}$ & 109.5 & $\mathrm{C} 42-\mathrm{C} 43-\mathrm{H} 43$ & 115.9 \\
\hline $\mathrm{N} 4-\mathrm{C} 5-\mathrm{H} 5 \mathrm{~B}$ & 109.5 & $\mathrm{C} 31-\mathrm{C} 43-\mathrm{H} 43$ & 115.9 \\
\hline
\end{tabular}




\begin{tabular}{|c|c|c|c|}
\hline $\mathrm{C} 6-\mathrm{C} 5-\mathrm{H} 5 \mathrm{~B}$ & 109.5 & $\mathrm{C} 32-\mathrm{C} 31-\mathrm{C} 36$ & $118.03(17)$ \\
\hline $\mathrm{H} 5 \mathrm{~A}-\mathrm{C} 5-\mathrm{H} 5 \mathrm{~B}$ & 108.1 & $\mathrm{C} 32-\mathrm{C} 31-\mathrm{C} 43$ & $118.94(16)$ \\
\hline $\mathrm{N} 1-\mathrm{C} 6-\mathrm{C} 5$ & $109.75(13)$ & $\mathrm{C} 36-\mathrm{C} 31-\mathrm{C} 43$ & $123.00(16)$ \\
\hline $\mathrm{N} 1-\mathrm{C} 6-\mathrm{H} 6 \mathrm{~A}$ & 109.7 & $\mathrm{C} 33-\mathrm{C} 32-\mathrm{C} 31$ & $121.50(18)$ \\
\hline $\mathrm{C} 5-\mathrm{C} 6-\mathrm{H} 6 \mathrm{~A}$ & 109.7 & $\mathrm{C} 33-\mathrm{C} 32-\mathrm{H} 32$ & 119.3 \\
\hline $\mathrm{N} 1-\mathrm{C} 6-\mathrm{H} 6 \mathrm{~B}$ & 109.7 & $\mathrm{C} 31-\mathrm{C} 32-\mathrm{H} 32$ & 119.3 \\
\hline $\mathrm{C} 5-\mathrm{C} 6-\mathrm{H} 6 \mathrm{~B}$ & 109.7 & $\mathrm{C} 32-\mathrm{C} 33-\mathrm{C} 34$ & $119.79(19)$ \\
\hline $\mathrm{H} 6 \mathrm{~A}-\mathrm{C} 6-\mathrm{H} 6 \mathrm{~B}$ & 108.2 & $\mathrm{C} 32-\mathrm{C} 33-\mathrm{H} 33$ & 120.1 \\
\hline $\mathrm{N} 1-\mathrm{C} 1-\mathrm{C} 21$ & $112.03(13)$ & $\mathrm{C} 34-\mathrm{C} 33-\mathrm{H} 33$ & 120.1 \\
\hline $\mathrm{N} 1-\mathrm{C} 1-\mathrm{C} 11$ & $110.05(13)$ & $\mathrm{C} 33-\mathrm{C} 34-\mathrm{C} 35$ & $119.62(19)$ \\
\hline $\mathrm{C} 21-\mathrm{C} 1-\mathrm{C} 11$ & $110.90(13)$ & $\mathrm{C} 33-\mathrm{C} 34-\mathrm{H} 34$ & 120.2 \\
\hline $\mathrm{N} 1-\mathrm{C} 1-\mathrm{H} 1$ & 107.9 & $\mathrm{C} 35-\mathrm{C} 34-\mathrm{H} 34$ & 120.2 \\
\hline $\mathrm{C} 21-\mathrm{C} 1-\mathrm{H} 1$ & 107.9 & $\mathrm{C} 36-\mathrm{C} 35-\mathrm{C} 34$ & $120.61(19)$ \\
\hline $\mathrm{C} 11-\mathrm{C} 1-\mathrm{H} 1$ & 107.9 & $\mathrm{C} 36-\mathrm{C} 35-\mathrm{H} 35$ & 119.7 \\
\hline $\mathrm{C} 16-\mathrm{C} 11-\mathrm{C} 12$ & $118.57(16)$ & $\mathrm{C} 34-\mathrm{C} 35-\mathrm{H} 35$ & 119.7 \\
\hline $\mathrm{C} 16-\mathrm{C} 11-\mathrm{C} 1$ & $121.68(14)$ & $\mathrm{C} 35-\mathrm{C} 36-\mathrm{C} 31$ & $120.44(18)$ \\
\hline $\mathrm{C} 12-\mathrm{C} 11-\mathrm{C} 1$ & $119.65(15)$ & $\mathrm{C} 35-\mathrm{C} 36-\mathrm{H} 36$ & 119.8 \\
\hline $\mathrm{C} 13-\mathrm{C} 12-\mathrm{C} 11$ & $121.12(16)$ & $\mathrm{C} 31-\mathrm{C} 36-\mathrm{H} 36$ & 119.8 \\
\hline $\mathrm{C} 13-\mathrm{C} 12-\mathrm{H} 12$ & 119.4 & $\mathrm{C} 56-\mathrm{N} 51-\mathrm{C} 52$ & $116.3(2)$ \\
\hline $\mathrm{C} 11-\mathrm{C} 12-\mathrm{H} 12$ & 119.4 & $\mathrm{~N} 51-\mathrm{C} 52-\mathrm{C} 53$ & $124.38(19)$ \\
\hline $\mathrm{C} 14-\mathrm{C} 13-\mathrm{C} 12$ & $117.95(17)$ & N51-C52-H52 & 117.8 \\
\hline $\mathrm{C} 14-\mathrm{C} 13-\mathrm{H} 13$ & 121.0 & $\mathrm{C} 53-\mathrm{C} 52-\mathrm{H} 52$ & 117.8 \\
\hline $\mathrm{C} 12-\mathrm{C} 13-\mathrm{H} 13$ & 121.0 & $\mathrm{C} 54-\mathrm{C} 53-\mathrm{C} 52$ & $117.59(17)$ \\
\hline $\mathrm{C} 13-\mathrm{C} 14-\mathrm{F} 14$ & $118.32(17)$ & $\mathrm{C} 54-\mathrm{C} 53-\mathrm{C} 57$ & $121.05(17)$ \\
\hline $\mathrm{C} 13-\mathrm{C} 14-\mathrm{C} 15$ & $123.42(17)$ & $\mathrm{C} 52-\mathrm{C} 53-\mathrm{C} 57$ & $121.35(16)$ \\
\hline $\mathrm{F} 14-\mathrm{C} 14-\mathrm{C} 15$ & $118.25(18)$ & $\mathrm{C} 55-\mathrm{C} 54-\mathrm{C} 53$ & $118.8(2)$ \\
\hline $\mathrm{C} 14-\mathrm{C} 15-\mathrm{C} 16$ & $117.93(17)$ & $\mathrm{C} 55-\mathrm{C} 54-\mathrm{H} 54$ & 120.6 \\
\hline $\mathrm{C} 14-\mathrm{C} 15-\mathrm{H} 15$ & 121.0 & $\mathrm{C} 53-\mathrm{C} 54-\mathrm{H} 54$ & 120.6 \\
\hline $\mathrm{C} 16-\mathrm{C} 15-\mathrm{H} 15$ & 121.0 & $\mathrm{C} 56-\mathrm{C} 55-\mathrm{C} 54$ & $118.9(2)$ \\
\hline $\mathrm{C} 11-\mathrm{C} 16-\mathrm{C} 15$ & $120.99(16)$ & C56-C55-H55 & 120.6 \\
\hline $\mathrm{C} 11-\mathrm{C} 16-\mathrm{H} 16$ & 119.5 & $\mathrm{C} 54-\mathrm{C} 55-\mathrm{H} 55$ & 120.6 \\
\hline $\mathrm{C} 15-\mathrm{C} 16-\mathrm{H} 16$ & 119.5 & N51-C56-C55 & $124.01(19)$ \\
\hline $\mathrm{C} 26-\mathrm{C} 21-\mathrm{C} 22$ & $118.54(16)$ & $\mathrm{N} 51-\mathrm{C} 56-\mathrm{H} 56$ & 118.0 \\
\hline $\mathrm{C} 26-\mathrm{C} 21-\mathrm{C} 1$ & $119.76(16)$ & $\mathrm{C} 55-\mathrm{C} 56-\mathrm{H} 56$ & 118.0 \\
\hline $\mathrm{C} 22-\mathrm{C} 21-\mathrm{C} 1$ & $121.69(15)$ & $\mathrm{O} 52-\mathrm{C} 57-\mathrm{O} 51$ & $125.05(17)$ \\
\hline $\mathrm{C} 23-\mathrm{C} 22-\mathrm{C} 21$ & $120.62(19)$ & $\mathrm{O} 52-\mathrm{C} 57-\mathrm{C} 53$ & $119.87(16)$ \\
\hline $\mathrm{C} 23-\mathrm{C} 22-\mathrm{H} 22$ & 119.7 & $\mathrm{O} 51-\mathrm{C} 57-\mathrm{C} 53$ & $115.07(15)$ \\
\hline $\mathrm{C} 6-\mathrm{N} 1-\mathrm{C} 2-\mathrm{C} 3$ & $-61.39(16)$ & $\mathrm{C} 21-\mathrm{C} 22-\mathrm{C} 23-\mathrm{C} 24$ & $0.3(3)$ \\
\hline $\mathrm{C} 1-\mathrm{N} 1-\mathrm{C} 2-\mathrm{C} 3$ & $174.41(13)$ & $\mathrm{C} 22-\mathrm{C} 23-\mathrm{C} 24-\mathrm{F} 24$ & $-178.91(18)$ \\
\hline $\mathrm{N} 1-\mathrm{C} 2-\mathrm{C} 3-\mathrm{N} 4$ & $58.60(17)$ & $\mathrm{C} 22-\mathrm{C} 23-\mathrm{C} 24-\mathrm{C} 25$ & $0.0(3)$ \\
\hline $\mathrm{C} 2-\mathrm{C} 3-\mathrm{N} 4-\mathrm{C} 5$ & $-54.81(17)$ & $\mathrm{F} 24-\mathrm{C} 24-\mathrm{C} 25-\mathrm{C} 26$ & $178.74(18)$ \\
\hline $\mathrm{C} 2-\mathrm{C} 3-\mathrm{N} 4-\mathrm{C} 41$ & $-176.42(14)$ & $\mathrm{C} 23-\mathrm{C} 24-\mathrm{C} 25-\mathrm{C} 26$ & $-0.1(3)$ \\
\hline $\mathrm{C} 3-\mathrm{N} 4-\mathrm{C} 5-\mathrm{C} 6$ & $55.99(17)$ & $\mathrm{C} 24-\mathrm{C} 25-\mathrm{C} 26-\mathrm{C} 21$ & $0.1(3)$ \\
\hline $\mathrm{C} 41-\mathrm{N} 4-\mathrm{C} 5-\mathrm{C} 6$ & $179.26(14)$ & $\mathrm{C} 22-\mathrm{C} 21-\mathrm{C} 26-\mathrm{C} 25$ & $0.1(3)$ \\
\hline $\mathrm{C} 2-\mathrm{N} 1-\mathrm{C} 6-\mathrm{C} 5$ & $61.43(16)$ & $\mathrm{C} 1-\mathrm{C} 21-\mathrm{C} 26-\mathrm{C} 25$ & $-179.35(16)$ \\
\hline $\mathrm{C} 1-\mathrm{N} 1-\mathrm{C} 6-\mathrm{C} 5$ & $-176.56(14)$ & $\mathrm{C} 5-\mathrm{N} 4-\mathrm{C} 41-\mathrm{C} 42$ & $-177.30(14)$ \\
\hline $\mathrm{N} 4-\mathrm{C} 5-\mathrm{C} 6-\mathrm{N} 1$ & $-60.11(17)$ & $\mathrm{C} 3-\mathrm{N} 4-\mathrm{C} 41-\mathrm{C} 42$ & $-55.58(19)$ \\
\hline
\end{tabular}




$\begin{array}{llll}\mathrm{C} 6-\mathrm{N} 1-\mathrm{C} 1-\mathrm{C} 21 & 44.09(18) & \mathrm{N} 4-\mathrm{C} 41-\mathrm{C} 42-\mathrm{C} 43 & 118.27(19) \\ \mathrm{C} 6-\mathrm{N} 1-\mathrm{C} 1-\mathrm{C} 11 & 167.96(13) & \mathrm{C} 41-\mathrm{C} 42-\mathrm{C} 43-\mathrm{C} 31 & 177.23(16) \\ \mathrm{C} 2-\mathrm{N} 1-\mathrm{C} 1-\mathrm{C} 11 & -70.92(16) & \mathrm{C} 42-\mathrm{C} 43-\mathrm{C} 31-\mathrm{C} 32 & -178.93(18) \\ \mathrm{C} 2-\mathrm{N} 1-\mathrm{C} 1-\mathrm{C} 21 & 165.22(13) & \mathrm{C} 42-\mathrm{C} 43-\mathrm{C} 31-\mathrm{C} 36 & -0.6(3) \\ \mathrm{N} 1-\mathrm{C} 1-\mathrm{C} 11-\mathrm{C} 16 & -33.4(2) & \mathrm{C} 36-\mathrm{C} 31-\mathrm{C} 32-\mathrm{C} 33 & -0.8(3) \\ \mathrm{C} 21-\mathrm{C} 1-\mathrm{C} 11-\mathrm{C} 16 & 91.08(18) & \mathrm{C} 43-\mathrm{C} 31-\mathrm{C} 32-\mathrm{C} 33 & 177.64(17) \\ \mathrm{N} 1-\mathrm{C} 1-\mathrm{C} 11-\mathrm{C} 12 & 150.17(15) & \mathrm{C} 31-\mathrm{C} 32-\mathrm{C} 33-\mathrm{C} 34 & 0.4(3) \\ \mathrm{C} 21-\mathrm{C} 1-\mathrm{C} 11-\mathrm{C} 12 & -85.31(18) & \mathrm{C} 32-\mathrm{C} 33-\mathrm{C} 34-\mathrm{C} 35 & 0.4(3) \\ \mathrm{C} 16-\mathrm{C} 11-\mathrm{C} 12-\mathrm{C} 13 & -0.3(2) & \mathrm{C} 33-\mathrm{C} 34-\mathrm{C} 35-\mathrm{C} 36 & -0.8(3) \\ \mathrm{C} 1-\mathrm{C} 11-\mathrm{C} 12-\mathrm{C} 13 & 176.25(15) & \mathrm{C} 34-\mathrm{C} 35-\mathrm{C} 36-\mathrm{C} 31 & 0.4(3) \\ \mathrm{C} 11-\mathrm{C} 12-\mathrm{C} 13-\mathrm{C} 14 & 1.3(3) & \mathrm{C} 32-\mathrm{C} 31-\mathrm{C} 36-\mathrm{C} 35 & 0.4(3) \\ \mathrm{C} 12-\mathrm{C} 13-\mathrm{C} 14-\mathrm{F} 14 & 177.30(15) & \mathrm{C} 43-\mathrm{C} 31-\mathrm{C} 36-\mathrm{C} 35 & -177.98(18) \\ \mathrm{C} 12-\mathrm{C} 13-\mathrm{C} 14-\mathrm{C} 15 & -1.7(3) & \mathrm{C} 56-\mathrm{N} 51-\mathrm{C} 52-\mathrm{C} 53 & 1.3(3) \\ \mathrm{C} 13-\mathrm{C} 14-\mathrm{C} 15-\mathrm{C} 16 & 1.0(3) & \mathrm{N} 51-\mathrm{C} 52-\mathrm{C} 53-\mathrm{C} 54 & -0.3(3) \\ \mathrm{F} 14-\mathrm{C} 14-\mathrm{C} 15-\mathrm{C} 16 & -177.96(16) & \mathrm{N} 51-\mathrm{C} 52-\mathrm{C} 53-\mathrm{C} 57 & 178.99(18) \\ \mathrm{C} 12-\mathrm{C} 11-\mathrm{C} 16-\mathrm{C} 15 & -0.4(3) & \mathrm{C} 52-\mathrm{C} 53-\mathrm{C} 54-\mathrm{C} 55 & -1.3(3) \\ \mathrm{C} 1-\mathrm{C} 11-\mathrm{C} 16-\mathrm{C} 15 & -176.87(15) & \mathrm{C} 57-\mathrm{C} 53-\mathrm{C} 54-\mathrm{C} 55 & 179.41(17) \\ \mathrm{C} 14-\mathrm{C} 15-\mathrm{C} 16-\mathrm{C} 11 & 0.1(3) & \mathrm{C} 53-\mathrm{C} 54-\mathrm{C} 55-\mathrm{C} 56 & 1.9(3) \\ \mathrm{N} 1-\mathrm{C} 1-\mathrm{C} 21-\mathrm{C} 26 & -111.11(17) & \mathrm{C} 52-\mathrm{N} 51-\mathrm{C} 56-\mathrm{C} 55 & -0.6(3) \\ \mathrm{C} 11-\mathrm{C} 1-\mathrm{C} 21-\mathrm{C} 26 & 125.50(17) & \mathrm{C} 54-\mathrm{C} 55-\mathrm{C} 56-\mathrm{N} 51 & -0.9(3) \\ \mathrm{N} 1-\mathrm{C} 1-\mathrm{C} 21-\mathrm{C} 22 & 69.4(2) & \mathrm{C} 54-\mathrm{C} 53-\mathrm{C} 57-\mathrm{O} 52 & 2.9(2) \\ \mathrm{C} 11-\mathrm{C} 1-\mathrm{C} 21-\mathrm{C} 22 & -54.0(2) & \mathrm{C} 52-\mathrm{C} 53-\mathrm{C} 57-\mathrm{O} 52 & -176.37(17) \\ \mathrm{C} 26-\mathrm{C} 21-\mathrm{C} 22-\mathrm{C} 23 & -0.3(3) & \mathrm{C} 54-\mathrm{C} 53-\mathrm{C} 57-\mathrm{O} 51 & -176.63(16) \\ \mathrm{C} 1-\mathrm{C} 21-\mathrm{C} 22-\mathrm{C} 23 & 179.18(16) & \mathrm{C} 52-\mathrm{C} 53-\mathrm{C} 57-\mathrm{O} 51 & 4.1(2) \\ - & & & \end{array}$

Hydrogen-bond geometry $\left(\AA,{ }^{\circ}\right)$

$C g 1$ and $C g 2$ represent the centroids of the $\mathrm{C} 11-\mathrm{C} 16$ and $\mathrm{C} 31-\mathrm{C} 36$ rings, respectively.

\begin{tabular}{lllll}
\hline$D-\mathrm{H} \cdots A$ & $D-\mathrm{H}$ & $\mathrm{H} \cdots A$ & $D \cdots A$ & $D-\mathrm{H} \cdots A$ \\
\hline $\mathrm{N} 4-\mathrm{H} 4 \cdots \mathrm{O} 51$ & 0.98 & 1.58 & $2.5630(19)$ & 175 \\
$\mathrm{C} 3-\mathrm{H} 3 B \cdots \mathrm{O} 52^{\mathrm{i}}$ & 0.99 & 2.53 & $3.440(2)$ & 153 \\
$\mathrm{C} 13-\mathrm{H} 13 \cdots \mathrm{O} 52^{\mathrm{ii}}$ & 0.95 & 2.57 & $3.442(2)$ & 152 \\
$\mathrm{C} 56-\mathrm{H} 56 \cdots \mathrm{O} 51^{\mathrm{iii}}$ & 0.95 & 2.34 & $3.236(3)$ & 158 \\
$\mathrm{C} 26-\mathrm{H} 26 \cdots C g 1^{\text {iv }}$ & 0.95 & 2.81 & $3.758(2)$ & 173 \\
$\mathrm{C} 55-\mathrm{H} 55 \cdots C g 2^{\mathrm{v}}$ & 0.95 & 2.85 & $3.567(2)$ & 133
\end{tabular}

Symmetry codes: (i) $x,-y+1, z+1 / 2$; (ii) $x-1,-y+1, z+1 / 2$; (iii) $x,-y+2, z-1 / 2$; (iv) $x,-y+1, z-1 / 2$; (v) $x, y, z-1$.

(II) 1-[Bis(4-fluorophenyl)methyl]-4-[(2E)-3-phenylprop-2-en-1-yl]piperazine-1,4-diium bis(4methylbenzenesulfonate) dihydrate

Crystal data

$\mathrm{C}_{26} \mathrm{H}_{28} \mathrm{~F}_{2} \mathrm{~N}_{2}{ }^{2+} \cdot 2 \mathrm{C}_{7} \mathrm{H}_{7} \mathrm{O}_{3} \mathrm{~S}^{-} \cdot 2 \mathrm{H}_{2} \mathrm{O}$

$M_{r}=784.91$

Monoclinic, $P 2_{1} / c$

$a=10.0546(5) \AA$

$b=14.8338(6) \AA$

$c=26.9437(12) \AA$

$\beta=106.497(3)^{\circ}$

$V=3853.2(3) \AA^{3}$
$Z=4$
$F(000)=1656$
$D_{\mathrm{x}}=1.353 \mathrm{Mg} \mathrm{m}^{-3}$

Mo $K \alpha$ radiation, $\lambda=0.71073 \AA$

Cell parameters from 9580 reflections

$\theta=1.6-28.4^{\circ}$ 
$\begin{aligned} \mu & =0.20 \mathrm{~mm}^{-1} \\ T & =200 \mathrm{~K}\end{aligned}$

Data collection

Bruker APEXII CCD area-detector diffractometer

$\varphi$ and $\omega$ scans

Absorption correction: multi-scan

(SADABS; Sheldrick, 2003)

$T_{\min }=0.877, T_{\max }=0.953$

45646 measured reflections

\section{Refinement}

Refinement on $F^{2}$

Least-squares matrix: full

$R\left[F^{2}>2 \sigma\left(F^{2}\right)\right]=0.075$

$w R\left(F^{2}\right)=0.202$

$S=1.09$

7212 reflections

527 parameters

27 restraints
Block, yellow

$0.59 \times 0.43 \times 0.24 \mathrm{~mm}$

7212 independent reflections

6219 reflections with $I>2 \sigma(I)$

$R_{\text {int }}=0.038$

$\theta_{\text {max }}=25.6^{\circ}, \theta_{\text {min }}=2.1^{\circ}$

$h=-12 \rightarrow 12$

$k=-15 \rightarrow 18$

$l=-32 \rightarrow 32$

Hydrogen site location: mixed

$\mathrm{H}$-atom parameters constrained

$w=1 /\left[\sigma^{2}\left(F_{\mathrm{o}}^{2}\right)+(0.0731 P)^{2}+8.0533 P\right]$

where $P=\left(F_{\mathrm{o}}^{2}+2 F_{\mathrm{c}}^{2}\right) / 3$

$(\Delta / \sigma)_{\max }=0.001$

$\Delta \rho_{\max }=0.71 \mathrm{e} \AA^{-3}$

$\Delta \rho_{\min }=-0.60$ e $\AA^{-3}$

\section{Special details}

Geometry. All e.s.d.'s (except the e.s.d. in the dihedral angle between two 1.s. planes) are estimated using the full covariance matrix. The cell e.s.d.'s are taken into account individually in the estimation of e.s.d.'s in distances, angles and torsion angles; correlations between e.s.d.'s in cell parameters are only used when they are defined by crystal symmetry. An approximate (isotropic) treatment of cell e.s.d.'s is used for estimating e.s.d.'s involving l.s. planes.

Fractional atomic coordinates and isotropic or equivalent isotropic displacement parameters $\left(\AA^{2}\right)$

\begin{tabular}{lllll}
\hline & $x$ & $y$ & $z$ & $U_{\text {iso }} * / U_{\text {eq }}$ \\
N1 & $0.9062(3)$ & $0.31590(17)$ & $0.06845(9)$ & $0.0283(5)$ \\
H1 & 0.9988 & 0.3286 & 0.0707 & $0.034^{*}$ \\
C2 & $0.8798(4)$ & $0.3563(2)$ & $0.11553(12)$ & $0.0377(8)$ \\
H2A & 0.9473 & 0.3313 & 0.1467 & $0.045^{*}$ \\
H2B & 0.7857 & 0.3391 & 0.1167 & $0.045^{*}$ \\
C3 & $0.8915(3)$ & $0.4569(2)$ & $0.11658(12)$ & $0.0329(7)$ \\
H3A & 0.8675 & 0.4802 & 0.1474 & $0.040^{*}$ \\
H3B & 0.9888 & 0.4740 & 0.1198 & $0.040^{*}$ \\
N4 & $0.7998(3)$ & $0.49940(19)$ & $0.06970(10)$ & $0.0374(6)$ \\
H4 & 0.7026 & 0.4838 & 0.0691 & $0.045^{*}$ \\
C5 & $0.8239(4)$ & $0.4592(2)$ & $0.02266(12)$ & $0.0376(8)$ \\
H5A & 0.9185 & 0.4751 & 0.0213 & $0.045^{*}$ \\
H5B & 0.7570 & 0.4853 & -0.0083 & $0.045^{*}$ \\
C6 & $0.8088(4)$ & $0.3587(2)$ & $0.02114(13)$ & $0.0421(8)$ \\
H6A & 0.8289 & 0.3351 & -0.0103 & $0.050^{*}$ \\
H6B & 0.7120 & 0.3426 & 0.0193 & $0.050^{*}$ \\
C1 & $0.8877(4)$ & $0.2133(2)$ & $0.06603(13)$ & $0.0376(8)$ \\
H1A & 0.7879 & 0.2008 & 0.0629 & $0.045^{*}$ \\
C11 & $0.9731(4)$ & $0.1704(2)$ & $0.11671(14)$ & $0.0411(8)$ \\
C12 & $0.9029(4)$ & $0.1445(3)$ & $0.15121(15)$ & $0.0464(9)$
\end{tabular}




\begin{tabular}{|c|c|c|c|c|}
\hline H12 & 0.8061 & 0.1555 & 0.1435 & $0.056^{*}$ \\
\hline $\mathrm{C} 13$ & $0.9716(5)$ & $0.1025(3)$ & $0.19714(16)$ & $0.0549(10)$ \\
\hline H13 & 0.9228 & 0.0848 & 0.2210 & $0.066^{*}$ \\
\hline $\mathrm{C} 14$ & $1.1094(5)$ & $0.0870(3)$ & $0.20764(16)$ & $0.0572(11)$ \\
\hline F14 & $1.1764(3)$ & $0.04445(19)$ & $0.25255(11)$ & $0.0858(10)$ \\
\hline $\mathrm{C} 15$ & $1.1850(5)$ & $0.1137(3)$ & $0.17454(19)$ & $0.0610(12)$ \\
\hline H15 & 1.2822 & 0.1039 & 0.1830 & $0.073^{*}$ \\
\hline $\mathrm{C} 16$ & $1.1131(4)$ & $0.1556(3)$ & $0.12814(16)$ & $0.0491(9)$ \\
\hline H16 & 1.1618 & 0.1740 & 0.1044 & $0.059 *$ \\
\hline $\mathrm{C} 21$ & $0.9177(4)$ & $0.1744(2)$ & $0.01823(13)$ & $0.0377(8)$ \\
\hline $\mathrm{C} 22$ & $1.0266(4)$ & $0.1995(3)$ & $-0.00073(16)$ & $0.0511(10)$ \\
\hline $\mathrm{H} 22$ & 1.0890 & 0.2450 & 0.0167 & $0.061^{*}$ \\
\hline $\mathrm{C} 23$ & $1.0464(5)$ & $0.1601(3)$ & -0.04430 & $0.0542(10)$ \\
\hline $\mathrm{H} 23$ & 1.1211 & 0.1780 & -0.0571 & $0.065^{*}$ \\
\hline $\mathrm{C} 24$ & $0.9575(5)$ & $0.0957(3)$ & $-0.06822(14)$ & $0.0503(10)$ \\
\hline F24 & $0.9777(4)$ & $0.05713(19)$ & $-0.11165(10)$ & $0.0848(10)$ \\
\hline $\mathrm{C} 25$ & $0.8505(5)$ & $0.0685(3)$ & $-0.05213(16)$ & $0.0588(11)$ \\
\hline $\mathrm{H} 25$ & 0.7905 & 0.0222 & -0.0701 & $0.071^{*}$ \\
\hline $\mathrm{C} 26$ & $0.8279(4)$ & $0.1087(3)$ & $-0.00872(16)$ & $0.0504(9)$ \\
\hline $\mathrm{H} 26$ & 0.7504 & 0.0910 & 0.0026 & $0.061^{*}$ \\
\hline $\mathrm{C} 41$ & $0.8089(4)$ & $0.5998(2)$ & $0.06908(14)$ & $0.0440(8)$ \\
\hline $\mathrm{H} 41 \mathrm{~A}$ & 0.9029 & 0.6170 & 0.0677 & $0.053^{*}$ \\
\hline H41B & 0.7418 & 0.6225 & 0.0371 & $0.053^{*}$ \\
\hline $\mathrm{C} 42$ & $0.7812(4)$ & $0.6449(2)$ & $0.11408(14)$ & $0.0432(8)$ \\
\hline $\mathrm{H} 42$ & 0.6939 & 0.6343 & 0.1201 & $0.052 *$ \\
\hline $\mathrm{C} 43$ & $0.8675(4)$ & $0.6978(3)$ & $0.14586(15)$ & $0.0457(9)$ \\
\hline $\mathrm{H} 43$ & 0.9579 & 0.7021 & 0.1416 & $0.055^{*}$ \\
\hline $\mathrm{C} 31$ & $0.8407(4)$ & $0.7523(3)$ & $0.18798(14)$ & $0.0442(8)$ \\
\hline $\mathrm{C} 32$ & $0.9371(5)$ & $0.8177(3)$ & $0.21029(16)$ & $0.0580(11)$ \\
\hline $\mathrm{H} 32$ & 1.0175 & 0.8247 & 0.1988 & $0.070^{*}$ \\
\hline $\mathrm{C} 33$ & $0.9191(6)$ & $0.8722(3)$ & $0.24818(18)$ & $0.0676(13)$ \\
\hline $\mathrm{H} 33$ & 0.9861 & 0.9174 & 0.2622 & $0.081^{*}$ \\
\hline $\mathrm{C} 34$ & $0.8081(6)$ & $0.8634(3)$ & $0.26634(17)$ & $0.0710(15)$ \\
\hline $\mathrm{H} 34$ & 0.7970 & 0.9018 & 0.2931 & $0.085^{*}$ \\
\hline $\mathrm{C} 35$ & $0.7098(5)$ & $0.7972(4)$ & $0.24544(19)$ & $0.0681(13)$ \\
\hline $\mathrm{H} 35$ & 0.6314 & 0.7895 & 0.2581 & $0.082 *$ \\
\hline $\mathrm{C} 36$ & $0.7275(4)$ & $0.7427(3)$ & $0.20607(16)$ & $0.0527(10)$ \\
\hline $\mathrm{H} 36$ & 0.6600 & 0.6981 & 0.1914 & $0.063^{*}$ \\
\hline C51 & $1.3052(3)$ & $0.6677(2)$ & $0.17881(13)$ & $0.0366(7)$ \\
\hline C52 & $1.2814(4)$ & $0.7583(3)$ & $0.16840(15)$ & $0.0446(8)$ \\
\hline $\mathrm{H} 52$ & 1.2474 & 0.7782 & 0.1336 & $0.054 *$ \\
\hline $\mathrm{C} 53$ & $1.3065(4)$ & $0.8197(3)$ & $0.20801(16)$ & $0.0507(9)$ \\
\hline H53 & 1.2896 & 0.8818 & 0.2003 & $0.061^{*}$ \\
\hline C54 & $1.3564(4)$ & $0.7923(3)$ & $0.25945(16)$ & $0.0504(9)$ \\
\hline $\mathrm{C} 55$ & $1.3787(5)$ & $0.7012(3)$ & $0.26940(15)$ & $0.0523(10)$ \\
\hline H55 & 1.4118 & 0.6810 & 0.3042 & $0.063^{*}$ \\
\hline C56 & $1.3537(4)$ & $0.6388(3)$ & $0.22950(14)$ & $0.0484(9)$ \\
\hline H56 & 1.3698 & 0.5765 & 0.2370 & $0.058^{*}$ \\
\hline
\end{tabular}




\begin{tabular}{|c|c|c|c|c|c|}
\hline C57 & $1.3887(6)$ & $0.8599(4)$ & $0.30303(19)$ & $0.0741(14)$ & \\
\hline H57A & 1.3970 & 0.8287 & 0.3358 & $0.111^{*}$ & \\
\hline H57B & 1.3139 & 0.9045 & 0.2970 & $0.111^{*}$ & \\
\hline $\mathrm{H} 57 \mathrm{C}$ & 1.4763 & 0.8904 & 0.3046 & $0.111^{*}$ & \\
\hline S51 & $1.27821(10)$ & $0.59086(6)$ & $0.12647(3)$ & $0.0400(2)$ & \\
\hline O51 & $1.2760(4)$ & $0.50202(19)$ & $0.14856(11)$ & $0.0621(8)$ & \\
\hline O52 & 1.3909 (3) & $0.60300(19)$ & $0.10373(12)$ & $0.0601(8)$ & \\
\hline O53 & $1.1460(3)$ & $0.6137(2)$ & $0.09039(10)$ & $0.0662(9)$ & \\
\hline C61 & $0.4319(4)$ & $0.1735(3)$ & $-0.00551(18)$ & $0.0413(11)$ & $0.832(6)$ \\
\hline C62 & $0.3808(17)$ & $0.0869(7)$ & -0.0143 & $0.093(2)$ & $0.832(6)$ \\
\hline $\mathrm{H} 62$ & 0.3444 & 0.0576 & 0.0104 & $0.112^{*}$ & $0.832(6)$ \\
\hline C63 & $0.3830(13)$ & $0.0428(6)$ & $-0.0596(4)$ & $0.092(4)$ & $0.832(6)$ \\
\hline H63 & 0.3522 & -0.0180 & -0.0645 & $0.110^{*}$ & $0.832(6)$ \\
\hline C64 & $0.4279(13)$ & $0.0839(6)$ & -0.0970 & $0.0534(13)$ & $0.832(6)$ \\
\hline C65 & $0.4758(6)$ & $0.1721(4)$ & $-0.0882(2)$ & $0.0484(15)$ & $0.832(6)$ \\
\hline $\mathrm{H} 65$ & 0.5067 & 0.2027 & -0.1138 & $0.058^{*}$ & $0.832(6)$ \\
\hline C66 & $0.4789(5)$ & $0.2158(3)$ & -0.04249 (19) & $0.0450(13)$ & $0.832(6)$ \\
\hline H66 & 0.5139 & 0.2755 & -0.0367 & $0.054 *$ & $0.832(6)$ \\
\hline C67 & $0.4262(16)$ & $0.0347(6)$ & $-0.1462(4)$ & $0.070(2)$ & $0.832(6)$ \\
\hline H67A & 0.4667 & 0.0732 & -0.1676 & $0.105^{*}$ & $0.832(6)$ \\
\hline H67B & 0.3303 & 0.0199 & -0.1652 & $0.105^{*}$ & $0.832(6)$ \\
\hline $\mathrm{H} 67 \mathrm{C}$ & 0.4804 & -0.0209 & -0.1376 & $0.105^{*}$ & $0.832(6)$ \\
\hline S61 & $0.44568(17)$ & $0.22444(13)$ & $0.05538(6)$ & $0.0442(4)$ & $0.832(6)$ \\
\hline O61 & $0.3748(4)$ & $0.3104(2)$ & $0.04318(14)$ & $0.0566(11)$ & $0.832(6)$ \\
\hline O62 & $0.3801(4)$ & $0.1655(3)$ & $0.08433(15)$ & $0.0681(12)$ & $0.832(6)$ \\
\hline O63 & $0.5873(5)$ & $0.2398(5)$ & $0.0767(2)$ & $0.097(2)$ & $0.832(6)$ \\
\hline C61A & $0.474(2)$ & $0.1737(10)$ & $-0.0054(6)$ & $0.0413(11)$ & $0.168(6)$ \\
\hline C62A & $0.397(8)$ & $0.095(3)$ & $-0.0121(15)$ & $0.093(2)$ & $0.168(6)$ \\
\hline H62A & 0.3742 & 0.0678 & 0.0164 & $0.112 *$ & $0.168(6)$ \\
\hline C63A & $0.353(7)$ & $0.056(4)$ & $-0.0611(16)$ & $0.092(4)$ & $0.168(6)$ \\
\hline H63A & 0.2792 & 0.0139 & -0.0684 & $0.110^{*}$ & $0.168(6)$ \\
\hline C64A & $0.413(7)$ & $0.078(3)$ & $-0.0988(13)$ & $0.0534(13)$ & $0.168(6)$ \\
\hline C65A & $0.502(4)$ & $0.152(2)$ & $-0.0898(8)$ & $0.0484(15)$ & $0.168(6)$ \\
\hline H65A & 0.5517 & 0.1670 & -0.1138 & $0.058^{*}$ & $0.168(6)$ \\
\hline C66A & $0.518(3)$ & $0.2049(15)$ & $-0.0460(7)$ & $0.0450(13)$ & $0.168(6)$ \\
\hline H66A & 0.5591 & 0.2628 & -0.0441 & $0.054^{*}$ & $0.168(6)$ \\
\hline C67A & $0.405(9)$ & 0.015 & $-0.1434(19)$ & $0.070(2)$ & $0.168(6)$ \\
\hline H67D & 0.3240 & 0.0304 & -0.1723 & $0.105^{*}$ & $0.168(6)$ \\
\hline H67E & 0.3960 & -0.0471 & -0.1324 & $0.105^{*}$ & $0.168(6)$ \\
\hline H67F & 0.4894 & 0.0206 & -0.1544 & $0.105^{*}$ & $0.168(6)$ \\
\hline S61A & $0.4738(9)$ & $0.2443(6)$ & $0.0478(3)$ & $0.0442(4)$ & $0.168(6)$ \\
\hline O61A & $0.4807(16)$ & $0.3360(7)$ & $0.0291(6)$ & $0.059(5)^{*}$ & $0.168(6)$ \\
\hline O62A & $0.3450(13)$ & $0.2298(11)$ & $0.0612(6)$ & $0.067(6)^{*}$ & $0.168(6)$ \\
\hline O63A & $0.5974(10)$ & $0.2273(9)$ & $0.0855(5)$ & $0.017(3)^{*}$ & $0.168(6)$ \\
\hline O71 & $1.1663(3)$ & 0.37595 (19) & $0.07577(12)$ & $0.0563(7)$ & \\
\hline H71A & 1.1873 & 0.4171 & 0.0985 & $0.084^{*}$ & \\
\hline H71B & 1.2430 & 0.3652 & 0.0700 & $0.090^{*}$ & \\
\hline O81 & $0.5371(3)$ & $0.4699(2)$ & 0.07130 & $0.0782(11)$ & \\
\hline
\end{tabular}




\begin{tabular}{lllll} 
H81B & 0.4924 & 0.5051 & 0.0850 & $0.117^{*}$ \\
$\mathrm{H} 81 \mathrm{~A}$ & 0.4824 & 0.4292 & 0.0563 & $0.117^{*}$ \\
\hline
\end{tabular}

Atomic displacement parameters $\left(A^{2}\right)$

\begin{tabular}{|c|c|c|c|c|c|c|}
\hline & $U^{11}$ & $U^{22}$ & $U^{33}$ & $U^{12}$ & $U^{13}$ & $U^{23}$ \\
\hline $\mathrm{N} 1$ & $0.0293(13)$ & $0.0278(13)$ & $0.0288(13)$ & $-0.0005(10)$ & $0.0099(10)$ & $-0.0034(10)$ \\
\hline $\mathrm{C} 2$ & $0.050(2)$ & $0.0381(18)$ & $0.0283(16)$ & $0.0069(15)$ & $0.0164(15)$ & $-0.0004(13)$ \\
\hline $\mathrm{C} 3$ & $0.0369(17)$ & $0.0332(17)$ & $0.0273(15)$ & $0.0053(13)$ & $0.0070(13)$ & $-0.0024(13)$ \\
\hline $\mathrm{N} 4$ & $0.0484(17)$ & $0.0359(15)$ & $0.0300(14)$ & $0.0117(13)$ & $0.0144(12)$ & $0.0002(12)$ \\
\hline $\mathrm{C} 5$ & $0.0432(19)$ & 0.0415 (19) & $0.0284(16)$ & $0.0103(15)$ & $0.0108(14)$ & $-0.0007(14)$ \\
\hline C6 & $0.049(2)$ & $0.044(2)$ & $0.0312(17)$ & $0.0087(16)$ & $0.0092(15)$ & $-0.0010(15)$ \\
\hline $\mathrm{C} 1$ & $0.0378(18)$ & $0.0334(18)$ & $0.0418(19)$ & $-0.0049(14)$ & $0.0114(15)$ & $-0.0050(14)$ \\
\hline $\mathrm{C} 11$ & $0.053(2)$ & $0.0281(17)$ & $0.0437(19)$ & $0.0048(15)$ & $0.0162(17)$ & $-0.0046(14)$ \\
\hline $\mathrm{C} 12$ & $0.056(2)$ & $0.039(2)$ & $0.047(2)$ & $0.0028(17)$ & $0.0188(18)$ & $0.0022(16)$ \\
\hline $\mathrm{C} 13$ & $0.076(3)$ & $0.042(2)$ & $0.049(2)$ & $0.002(2)$ & $0.022(2)$ & $0.0085(18)$ \\
\hline $\mathrm{C} 14$ & $0.082(3)$ & $0.033(2)$ & $0.045(2)$ & $0.003(2)$ & $0.001(2)$ & $0.0006(17)$ \\
\hline F14 & $0.110(2)$ & $0.0607(17)$ & $0.0622(16)$ & $0.0123(16)$ & $-0.0149(16)$ & $0.0141(13)$ \\
\hline $\mathrm{C} 15$ & $0.046(2)$ & $0.045(2)$ & $0.083(3)$ & $0.0035(18)$ & $0.004(2)$ & $-0.012(2)$ \\
\hline $\mathrm{C} 16$ & $0.056(2)$ & $0.037(2)$ & $0.056(2)$ & $-0.0032(17)$ & $0.0189(19)$ & $-0.0028(17)$ \\
\hline $\mathrm{C} 21$ & $0.049(2)$ & $0.0317(17)$ & $0.0335(17)$ & $0.0078(15)$ & $0.0142(15)$ & -0.0001 \\
\hline $\mathrm{C} 22$ & $0.056(2)$ & $0.047(2)$ & $0.055(2)$ & $-0.0161(18)$ & $0.0226(19)$ & $-0.0180(18)$ \\
\hline $\mathrm{C} 23$ & $0.074(3)$ & $0.049(2)$ & $0.052(2)$ & $-0.002(2)$ & $0.037(2)$ & $-0.0044(18)$ \\
\hline $\mathrm{C} 24$ & $0.079(3)$ & $0.037(2)$ & $0.0364(19)$ & $0.0094(19)$ & $0.0184(19)$ & $-0.0044(15)$ \\
\hline F24 & $0.150(3)$ & $0.0631(17)$ & $0.0510(15)$ & $-0.0019(18)$ & $0.0443(17)$ & $-0.0222(13)$ \\
\hline $\mathrm{C} 25$ & $0.075(3)$ & $0.044(2)$ & $0.052(2)$ & $-0.015(2)$ & 0.009 (2) & $-0.0171(19)$ \\
\hline $\mathrm{C} 26$ & $0.053(2)$ & $0.040(2)$ & $0.061(2)$ & $-0.0046(17)$ & $0.022(2)$ & $0.0034(18)$ \\
\hline $\mathrm{C} 41$ & $0.056(2)$ & $0.0346(18)$ & $0.0409(19)$ & $0.0125(16)$ & $0.0140(17)$ & $0.0048(15)$ \\
\hline $\mathrm{C} 42$ & $0.051(2)$ & $0.0348(19)$ & $0.045(2)$ & $0.0131(16)$ & $0.0159(17)$ & $0.0001(15)$ \\
\hline C43 & $0.044(2)$ & $0.045(2)$ & $0.051(2)$ & $0.0082(17)$ & $0.0189(17)$ & $0.0088(17)$ \\
\hline $\mathrm{C} 31$ & $0.053(2)$ & $0.042(2)$ & $0.0365(18)$ & $0.0099(17)$ & $0.0110(16)$ & $0.0059(15)$ \\
\hline $\mathrm{C} 32$ & $0.063(3)$ & $0.062(3)$ & $0.050(2)$ & $-0.002(2)$ & $0.016(2)$ & $0.010(2)$ \\
\hline $\mathrm{C} 33$ & $0.090(4)$ & $0.050(3)$ & $0.051(2)$ & $-0.011(2)$ & $0.001(2)$ & $0.000(2)$ \\
\hline $\mathrm{C} 34$ & $0.112(4)$ & $0.056(3)$ & $0.040(2)$ & $0.031(3)$ & $0.013(3)$ & $-0.006(2)$ \\
\hline $\mathrm{C} 35$ & $0.064(3)$ & $0.083(3)$ & $0.065(3)$ & $0.027(3)$ & $0.032(2)$ & $0.016(3)$ \\
\hline $\mathrm{C} 36$ & $0.053(2)$ & $0.048(2)$ & $0.054(2)$ & $0.0001(18)$ & $0.0090(19)$ & $0.0028(18)$ \\
\hline $\mathrm{C} 51$ & $0.0362(17)$ & $0.0393(18)$ & $0.0359(17)$ & $0.0018(14)$ & $0.0130(14)$ & $0.0037(14)$ \\
\hline $\mathrm{C} 52$ & $0.052(2)$ & $0.041(2)$ & $0.0402(19)$ & $0.0053(16)$ & $0.0121(16)$ & $0.0039(16)$ \\
\hline $\mathrm{C} 53$ & $0.056(2)$ & $0.040(2)$ & $0.059(2)$ & $0.0058(17)$ & $0.022(2)$ & $0.0019(18)$ \\
\hline $\mathrm{C} 54$ & $0.054(2)$ & $0.054(2)$ & $0.049(2)$ & $-0.0008(18)$ & $0.0243(18)$ & $-0.0107(18)$ \\
\hline $\mathrm{C} 55$ & $0.065(3)$ & $0.059(2)$ & $0.0348(19)$ & $-0.005(2)$ & $0.0172(18)$ & $0.0042(17)$ \\
\hline $\mathrm{C} 56$ & $0.064(2)$ & $0.044(2)$ & $0.0393(19)$ & $-0.0007(18)$ & $0.0185(18)$ & $0.0058(16)$ \\
\hline $\mathrm{C} 57$ & $0.095(4)$ & $0.071(3)$ & $0.061(3)$ & $-0.003(3)$ & $0.030(3)$ & $-0.019(2)$ \\
\hline S51 & $0.0485(5)$ & $0.0403(5)$ & $0.0339(4)$ & -0.0020 & $0.0159(4)$ & $0.0013(3)$ \\
\hline O51 & $0.100(2)$ & $0.0417(15)$ & $0.0421(15)$ & $-0.0197(15)$ & $0.0159(15)$ & $-0.0003(12)$ \\
\hline O52 & $0.0704(19)$ & $0.0503(17)$ & $0.076(2)$ & $-0.0065(14)$ & $0.0471(17)$ & $-0.0141(14)$ \\
\hline $\mathrm{O} 53$ & $0.0642(19)$ & $0.093(2)$ & $0.0365(14)$ & $0.0181(17)$ & $0.0066(13)$ & $-0.0071(15)$ \\
\hline C61 & $0.021(3)$ & $0.045(2)$ & $0.060(2)$ & $0.0073(18)$ & $0.0144(19)$ & $-0.0026(18)$ \\
\hline
\end{tabular}


supporting information

\begin{tabular}{lllllll} 
C62 & $0.153(7)$ & $0.058(4)$ & $0.100(4)$ & $-0.043(4)$ & $0.088(5)$ & $-0.025(3)$ \\
C63 & $0.160(9)$ & $0.046(4)$ & $0.094(4)$ & $-0.028(6)$ & $0.076(5)$ & $-0.019(3)$ \\
C64 & $0.057(4)$ & $0.050(3)$ & $0.053(2)$ & $0.013(2)$ & $0.014(2)$ & $0.0022(19)$ \\
C65 & $0.040(3)$ & $0.057(4)$ & $0.044(2)$ & $0.006(3)$ & $0.0053(19)$ & $0.011(2)$ \\
C66 & $0.030(3)$ & $0.044(2)$ & $0.054(2)$ & $0.001(2)$ & $-0.001(2)$ & $0.0033(19)$ \\
C67 & $0.093(7)$ & $0.062(6)$ & $0.054(3)$ & $0.010(4)$ & $0.018(3)$ & $0.000(4)$ \\
S61 & $0.0298(7)$ & $0.0456(8)$ & $0.0606(7)$ & $-0.0028(6)$ & $0.0183(5)$ & $-0.0034(6)$ \\
O61 & $0.058(2)$ & $0.053(2)$ & $0.060(2)$ & $0.0118(16)$ & $0.0186(17)$ & $-0.0062(16)$ \\
O62 & $0.072(3)$ & $0.073(3)$ & $0.068(2)$ & $0.000(2)$ & $0.034(2)$ & $0.008(2)$ \\
O63 & $0.068(3)$ & $0.138(6)$ & $0.091(4)$ & $0.017(3)$ & $0.034(3)$ & $-0.017(4)$ \\
C61A & $0.021(3)$ & $0.045(2)$ & $0.060(2)$ & $0.0073(18)$ & $0.0144(19)$ & $-0.0026(18)$ \\
C62A & $0.153(7)$ & $0.058(4)$ & $0.100(4)$ & $-0.043(4)$ & $0.088(5)$ & $-0.025(3)$ \\
C63A & $0.160(9)$ & $0.046(4)$ & $0.094(4)$ & $-0.028(6)$ & $0.076(5)$ & $-0.019(3)$ \\
C64A & $0.057(4)$ & $0.050(3)$ & $0.053(2)$ & $0.013(2)$ & $0.014(2)$ & $0.0022(19)$ \\
C65A & $0.040(3)$ & $0.057(4)$ & $0.044(2)$ & $0.006(3)$ & $0.0053(19)$ & $0.011(2)$ \\
C66A & $0.030(3)$ & $0.044(2)$ & $0.054(2)$ & $0.001(2)$ & $-0.001(2)$ & $0.0033(19)$ \\
C67A & $0.093(7)$ & $0.062(6)$ & $0.054(3)$ & $0.010(4)$ & $0.018(3)$ & $0.000(4)$ \\
S61A & $0.0298(7)$ & $0.0456(8)$ & $0.0606(7)$ & $-0.0028(6)$ & $0.0183(5)$ & $-0.0034(6)$ \\
O71 & $0.0462(15)$ & $0.0511(16)$ & $0.0750(19)$ & $-0.0082(12)$ & $0.0228(14)$ & $-0.0225(14)$ \\
O81 & $0.0590(19)$ & $0.065(2)$ & $0.128(3)$ & $0.0021(16)$ & $0.056(2)$ & $-0.015(2)$ \\
& & & & & & \\
\hline
\end{tabular}

Geometric parameters $\left(\AA,{ }^{o}\right)$

\begin{tabular}{llll}
\hline $\mathrm{N} 1-\mathrm{C} 2$ & $1.493(4)$ & $\mathrm{C} 36-\mathrm{H} 36$ & 0.9500 \\
$\mathrm{~N} 1-\mathrm{C} 6$ & $1.509(4)$ & $\mathrm{C} 51-\mathrm{C} 52$ & $1.380(5)$ \\
$\mathrm{N} 1-\mathrm{C} 1$ & $1.532(4)$ & $\mathrm{C} 51-\mathrm{C} 56$ & $1.382(5)$ \\
$\mathrm{N} 1-\mathrm{H} 1$ & 0.9355 & $\mathrm{C} 51-\mathrm{S} 51$ & $1.773(4)$ \\
$\mathrm{C} 2-\mathrm{C} 3$ & $1.497(5)$ & $\mathrm{C} 52-\mathrm{C} 53$ & $1.371(5)$ \\
$\mathrm{C} 2-\mathrm{H} 2 \mathrm{~A}$ & 0.9900 & $\mathrm{C} 52-\mathrm{H} 52$ & 0.9500 \\
$\mathrm{C} 2-\mathrm{H} 2 \mathrm{~B}$ & 0.9900 & $\mathrm{C} 53-\mathrm{C} 54$ & $1.394(6)$ \\
$\mathrm{C} 3-\mathrm{N} 4$ & $1.477(4)$ & $\mathrm{C} 53-\mathrm{H} 53$ & 0.9500 \\
$\mathrm{C} 3-\mathrm{H} 3 \mathrm{~A}$ & 0.9900 & $\mathrm{C} 54-\mathrm{C} 55$ & $1.384(6)$ \\
$\mathrm{C} 3-\mathrm{H} 3 \mathrm{~B}$ & 0.9900 & $\mathrm{C} 54-\mathrm{C} 57$ & $1.507(6)$ \\
$\mathrm{N} 4-\mathrm{C} 5$ & $1.482(4)$ & $\mathrm{C} 55-\mathrm{C} 56$ & $1.387(6)$ \\
$\mathrm{N} 4-\mathrm{C} 41$ & $1.493(4)$ & $\mathrm{C} 55-\mathrm{H} 55$ & 0.9500 \\
$\mathrm{~N} 4-\mathrm{H} 4$ & 0.9998 & $\mathrm{C} 56-\mathrm{H} 56$ & 0.9500 \\
$\mathrm{C} 5-\mathrm{C} 6$ & $1.497(5)$ & $\mathrm{C} 57-\mathrm{H} 57 \mathrm{~A}$ & 0.9800 \\
$\mathrm{C} 5-\mathrm{H} 5 \mathrm{~A}$ & 0.9900 & $\mathrm{C} 57-\mathrm{H} 57 \mathrm{~B}$ & 0.9800 \\
$\mathrm{C} 5-\mathrm{H} 5 \mathrm{~B}$ & 0.9900 & $\mathrm{C} 57-\mathrm{H} 57 \mathrm{C}$ & 0.9800 \\
$\mathrm{C} 6-\mathrm{H} 6 \mathrm{~A}$ & 0.9900 & $\mathrm{~S} 51-\mathrm{O} 52$ & $1.444(3)$ \\
$\mathrm{C} 6-\mathrm{H} 6 \mathrm{~B}$ & 0.9900 & $\mathrm{~S} 51-\mathrm{O} 53$ & $1.446(3)$ \\
$\mathrm{C} 1-\mathrm{C} 21$ & $1.518(5)$ & $\mathrm{S} 51-\mathrm{O} 51$ & $1.449(3)$ \\
$\mathrm{C} 1-\mathrm{C} 11$ & $1.531(5)$ & $\mathrm{C} 61-\mathrm{C} 66$ & $1.370(6)$ \\
$\mathrm{C} 1-\mathrm{H} 1 \mathrm{~A}$ & 1.0000 & $\mathrm{C} 61-\mathrm{C} 62$ & $1.379(7)$ \\
$\mathrm{C} 11-\mathrm{C} 16$ & $1.370(5)$ & $\mathrm{C} 61-\mathrm{S} 61$ & $1.776(4)$ \\
$\mathrm{C} 11-\mathrm{C} 12$ & $1.372(5)$ & $\mathrm{C} 62-\mathrm{C} 63$ & $1.390(8)$ \\
$\mathrm{C} 12-\mathrm{C} 13$ & $1.382(6)$ & $\mathrm{C} 62-\mathrm{H} 62$ & 0.9500 \\
$\mathrm{C} 12-\mathrm{H} 12$ & 0.9500 & $\mathrm{C} 63-\mathrm{C} 64$ & $1.361(7)$ \\
& & &
\end{tabular}




\begin{tabular}{|c|c|c|c|}
\hline $\mathrm{C} 13-\mathrm{C} 14$ & $1.352(7)$ & $\mathrm{C} 63-\mathrm{H} 63$ & 0.9500 \\
\hline C13-H13 & 0.9500 & C64-C65 & $1.390(7)$ \\
\hline C14-F14 & $1.361(5)$ & C64-C67 & $1.508(6)$ \\
\hline $\mathrm{C} 14-\mathrm{C} 15$ & $1.384(7)$ & C65-C66 & $1.384(7)$ \\
\hline $\mathrm{C} 15-\mathrm{C} 16$ & $1.400(6)$ & $\mathrm{C} 65-\mathrm{H} 65$ & 0.9500 \\
\hline $\mathrm{C} 15-\mathrm{H} 15$ & 0.9500 & C66-H66 & 0.9500 \\
\hline $\mathrm{C} 16-\mathrm{H} 16$ & 0.9500 & C67-H67A & 0.9800 \\
\hline $\mathrm{C} 21-\mathrm{C} 22$ & $1.384(5)$ & C67-H67B & 0.9800 \\
\hline $\mathrm{C} 21-\mathrm{C} 26$ & $1.386(5)$ & C67-H67C & 0.9800 \\
\hline $\mathrm{C} 22-\mathrm{C} 23$ & $1.376(5)$ & $\mathrm{S} 61-\mathrm{O} 63$ & $1.395(5)$ \\
\hline $\mathrm{C} 22-\mathrm{H} 22$ & 0.9500 & $\mathrm{~S} 61-\mathrm{O} 62$ & $1.448(4)$ \\
\hline $\mathrm{C} 23-\mathrm{C} 24$ & $1.341(6)$ & S61-O61 & $1.452(4)$ \\
\hline $\mathrm{C} 23-\mathrm{H} 23$ & 0.9500 & $\mathrm{C} 61 \mathrm{~A}-\mathrm{C} 66 \mathrm{~A}$ & $1.373(7)$ \\
\hline $\mathrm{C} 24-\mathrm{C} 25$ & $1.332(6)$ & $\mathrm{C} 61 \mathrm{~A}-\mathrm{C} 62 \mathrm{~A}$ & $1.383(8)$ \\
\hline $\mathrm{C} 24-\mathrm{F} 24$ & $1.368(4)$ & C61A-S61A & $1.773(6)$ \\
\hline $\mathrm{C} 25-\mathrm{C} 26$ & $1.388(6)$ & $\mathrm{C} 62 \mathrm{~A}-\mathrm{C} 63 \mathrm{~A}$ & $1.392(10)$ \\
\hline $\mathrm{C} 25-\mathrm{H} 25$ & 0.9500 & $\mathrm{C} 62 \mathrm{~A}-\mathrm{H} 62 \mathrm{~A}$ & 0.9500 \\
\hline $\mathrm{C} 26-\mathrm{H} 26$ & 0.9500 & $\mathrm{C} 63 \mathrm{~A}-\mathrm{C} 64 \mathrm{~A}$ & $1.362(8)$ \\
\hline $\mathrm{C} 41-\mathrm{C} 42$ & $1.479(5)$ & $\mathrm{C} 63 \mathrm{~A}-\mathrm{H} 63 \mathrm{~A}$ & 0.9500 \\
\hline $\mathrm{C} 41-\mathrm{H} 41 \mathrm{~A}$ & 0.9900 & $\mathrm{C} 64 \mathrm{~A}-\mathrm{C} 65 \mathrm{~A}$ & $1.392(9)$ \\
\hline $\mathrm{C} 41-\mathrm{H} 41 \mathrm{~B}$ & 0.9900 & $\mathrm{C} 64 \mathrm{~A}-\mathrm{C} 67 \mathrm{~A}$ & $1.509(8)$ \\
\hline $\mathrm{C} 42-\mathrm{C} 43$ & $1.297(6)$ & $\mathrm{C} 65 \mathrm{~A}-\mathrm{C} 66 \mathrm{~A}$ & $1.384(8)$ \\
\hline $\mathrm{C} 42-\mathrm{H} 42$ & 0.9500 & $\mathrm{C} 65 \mathrm{~A}-\mathrm{H} 65 \mathrm{~A}$ & 0.9500 \\
\hline $\mathrm{C} 43-\mathrm{C} 31$ & $1.479(5)$ & $\mathrm{C} 66 \mathrm{~A}-\mathrm{H} 66 \mathrm{~A}$ & 0.9500 \\
\hline $\mathrm{C} 43-\mathrm{H} 43$ & 0.9500 & C67A-H67D & 0.9800 \\
\hline $\mathrm{C} 31-\mathrm{C} 36$ & $1.367(6)$ & C67A-H67E & 0.9800 \\
\hline $\mathrm{C} 31-\mathrm{C} 32$ & $1.382(6)$ & $\mathrm{C} 67 \mathrm{~A}-\mathrm{H} 67 \mathrm{~F}$ & 0.9800 \\
\hline $\mathrm{C} 32-\mathrm{C} 33$ & $1.353(7)$ & $\mathrm{S} 61 \mathrm{~A}-\mathrm{O} 63 \mathrm{~A}$ & $1.388(6)$ \\
\hline C $32-\mathrm{H} 32$ & 0.9500 & $\mathrm{~S} 61 \mathrm{~A}-\mathrm{O} 62 \mathrm{~A}$ & $1.456(6)$ \\
\hline C33-C34 & $1.346(8)$ & $\mathrm{S} 61 \mathrm{~A}-\mathrm{O} 61 \mathrm{~A}$ & $1.458(6)$ \\
\hline C $33-\mathrm{H} 33$ & 0.9500 & O71-H71A & 0.8475 \\
\hline $\mathrm{C} 34-\mathrm{C} 35$ & $1.394(8)$ & O71-H71B & 0.8448 \\
\hline C $34-\mathrm{H} 34$ & 0.9500 & O81-H81B & 0.8387 \\
\hline $\mathrm{C} 35-\mathrm{C} 36$ & $1.384(6)$ & O81-H81A & 0.8393 \\
\hline $\mathrm{C} 35-\mathrm{H} 35$ & 0.9500 & & \\
\hline $\mathrm{C} 2-\mathrm{N} 1-\mathrm{C} 6$ & $108.6(2)$ & $\mathrm{C} 33-\mathrm{C} 34-\mathrm{H} 34$ & 120.4 \\
\hline $\mathrm{C} 2-\mathrm{N} 1-\mathrm{C} 1$ & $112.7(2)$ & $\mathrm{C} 35-\mathrm{C} 34-\mathrm{H} 34$ & 120.4 \\
\hline $\mathrm{C} 6-\mathrm{N} 1-\mathrm{C} 1$ & $109.9(2)$ & $\mathrm{C} 36-\mathrm{C} 35-\mathrm{C} 34$ & $119.3(4)$ \\
\hline $\mathrm{C} 2-\mathrm{N} 1-\mathrm{H} 1$ & 106.2 & $\mathrm{C} 36-\mathrm{C} 35-\mathrm{H} 35$ & 120.3 \\
\hline $\mathrm{C} 6-\mathrm{N} 1-\mathrm{H} 1$ & 111.4 & $\mathrm{C} 34-\mathrm{C} 35-\mathrm{H} 35$ & 120.3 \\
\hline $\mathrm{C} 1-\mathrm{N} 1-\mathrm{H} 1$ & 108.0 & $\mathrm{C} 31-\mathrm{C} 36-\mathrm{C} 35$ & $120.9(4)$ \\
\hline $\mathrm{N} 1-\mathrm{C} 2-\mathrm{C} 3$ & $112.6(3)$ & $\mathrm{C} 31-\mathrm{C} 36-\mathrm{H} 36$ & 119.5 \\
\hline $\mathrm{N} 1-\mathrm{C} 2-\mathrm{H} 2 \mathrm{~A}$ & 109.1 & $\mathrm{C} 35-\mathrm{C} 36-\mathrm{H} 36$ & 119.5 \\
\hline $\mathrm{C} 3-\mathrm{C} 2-\mathrm{H} 2 \mathrm{~A}$ & 109.1 & $\mathrm{C} 52-\mathrm{C} 51-\mathrm{C} 56$ & $119.7(3)$ \\
\hline $\mathrm{N} 1-\mathrm{C} 2-\mathrm{H} 2 \mathrm{~B}$ & 109.1 & $\mathrm{C} 52-\mathrm{C} 51-\mathrm{S} 51$ & $119.1(3)$ \\
\hline $\mathrm{C} 3-\mathrm{C} 2-\mathrm{H} 2 \mathrm{~B}$ & 109.1 & $\mathrm{C} 56-\mathrm{C} 51-\mathrm{S} 51$ & $121.2(3)$ \\
\hline $\mathrm{H} 2 \mathrm{~A}-\mathrm{C} 2-\mathrm{H} 2 \mathrm{~B}$ & 107.8 & $\mathrm{C} 53-\mathrm{C} 52-\mathrm{C} 51$ & $120.4(3)$ \\
\hline
\end{tabular}




\begin{tabular}{|c|c|}
\hline $\mathrm{N} 4-\mathrm{C} 3-\mathrm{C} 2$ & $112.5(3)$ \\
\hline $\mathrm{N} 4-\mathrm{C} 3-\mathrm{H} 3 \mathrm{~A}$ & 109.1 \\
\hline $\mathrm{C} 2-\mathrm{C} 3-\mathrm{H} 3 \mathrm{~A}$ & 109.1 \\
\hline $\mathrm{N} 4-\mathrm{C} 3-\mathrm{H} 3 \mathrm{~B}$ & 109.1 \\
\hline $\mathrm{C} 2-\mathrm{C} 3-\mathrm{H} 3 \mathrm{~B}$ & 109.1 \\
\hline $\mathrm{H} 3 \mathrm{~A}-\mathrm{C} 3-\mathrm{H} 3 \mathrm{~B}$ & 107.8 \\
\hline $\mathrm{C} 3-\mathrm{N} 4-\mathrm{C} 5$ & $110.2(2)$ \\
\hline $\mathrm{C} 3-\mathrm{N} 4-\mathrm{C} 41$ & $114.2(3)$ \\
\hline $\mathrm{C} 5-\mathrm{N} 4-\mathrm{C} 41$ & $111.5(3)$ \\
\hline $\mathrm{C} 3-\mathrm{N} 4-\mathrm{H} 4$ & 106.5 \\
\hline $\mathrm{C} 5-\mathrm{N} 4-\mathrm{H} 4$ & 106.9 \\
\hline $\mathrm{C} 41-\mathrm{N} 4-\mathrm{H} 4$ & 107.0 \\
\hline $\mathrm{N} 4-\mathrm{C} 5-\mathrm{C} 6$ & $112.6(3)$ \\
\hline $\mathrm{N} 4-\mathrm{C} 5-\mathrm{H} 5 \mathrm{~A}$ & 109.1 \\
\hline $\mathrm{C} 6-\mathrm{C} 5-\mathrm{H} 5 \mathrm{~A}$ & 109.1 \\
\hline $\mathrm{N} 4-\mathrm{C} 5-\mathrm{H} 5 \mathrm{~B}$ & 109.1 \\
\hline $\mathrm{C} 6-\mathrm{C} 5-\mathrm{H} 5 \mathrm{~B}$ & 109.1 \\
\hline $\mathrm{H} 5 \mathrm{~A}-\mathrm{C} 5-\mathrm{H} 5 \mathrm{~B}$ & 107.8 \\
\hline $\mathrm{C} 5-\mathrm{C} 6-\mathrm{N} 1$ & $111.1(3)$ \\
\hline $\mathrm{C} 5-\mathrm{C} 6-\mathrm{H} 6 \mathrm{~A}$ & 109.4 \\
\hline $\mathrm{N} 1-\mathrm{C} 6-\mathrm{H} 6 \mathrm{~A}$ & 109.4 \\
\hline $\mathrm{C} 5-\mathrm{C} 6-\mathrm{H} 6 \mathrm{~B}$ & 109.4 \\
\hline $\mathrm{N} 1-\mathrm{C} 6-\mathrm{H} 6 \mathrm{~B}$ & 109.4 \\
\hline $\mathrm{H} 6 \mathrm{~A}-\mathrm{C} 6-\mathrm{H} 6 \mathrm{~B}$ & 108.0 \\
\hline $\mathrm{C} 21-\mathrm{C} 1-\mathrm{C} 11$ & $113.7(3)$ \\
\hline $\mathrm{C} 21-\mathrm{C} 1-\mathrm{N} 1$ & 111.3 \\
\hline $\mathrm{C} 11-\mathrm{C} 1-\mathrm{N} 1$ & $110.2(3)$ \\
\hline $\mathrm{C} 21-\mathrm{C} 1-\mathrm{H} 1 \mathrm{~A}$ & 107.1 \\
\hline $\mathrm{C} 11-\mathrm{C} 1-\mathrm{H} 1 \mathrm{~A}$ & 107.1 \\
\hline $\mathrm{N} 1-\mathrm{C} 1-\mathrm{H} 1 \mathrm{~A}$ & 107.1 \\
\hline $\mathrm{C} 16-\mathrm{C} 11-\mathrm{C} 12$ & $119.8(4)$ \\
\hline $\mathrm{C} 16-\mathrm{C} 11-\mathrm{C} 1$ & $123.2(3)$ \\
\hline $\mathrm{C} 12-\mathrm{C} 11-\mathrm{C} 1$ & $117.0(3)$ \\
\hline $\mathrm{C} 11-\mathrm{C} 12-\mathrm{C} 13$ & $120.7(4)$ \\
\hline $\mathrm{C} 11-\mathrm{C} 12-\mathrm{H} 12$ & 119.7 \\
\hline $\mathrm{C} 13-\mathrm{C} 12-\mathrm{H} 12$ & 119.7 \\
\hline $\mathrm{C} 14-\mathrm{C} 13-\mathrm{C} 12$ & $119.1(4)$ \\
\hline $\mathrm{C} 14-\mathrm{C} 13-\mathrm{H} 13$ & 120.5 \\
\hline $\mathrm{C} 12-\mathrm{C} 13-\mathrm{H} 13$ & 120.5 \\
\hline $\mathrm{C} 13-\mathrm{C} 14-\mathrm{F} 14$ & $118.9(4)$ \\
\hline $\mathrm{C} 13-\mathrm{C} 14-\mathrm{C} 15$ & $122.3(4)$ \\
\hline $\mathrm{F} 14-\mathrm{C} 14-\mathrm{C} 15$ & $118.8(4)$ \\
\hline $\mathrm{C} 14-\mathrm{C} 15-\mathrm{C} 16$ & $117.6(4)$ \\
\hline $\mathrm{C} 14-\mathrm{C} 15-\mathrm{H} 15$ & 121.2 \\
\hline $\mathrm{C} 16-\mathrm{C} 15-\mathrm{H} 15$ & 121.2 \\
\hline $\mathrm{C} 11-\mathrm{C} 16-\mathrm{C} 15$ & $120.6(4)$ \\
\hline $\mathrm{C} 11-\mathrm{C} 16-\mathrm{H} 16$ & 119.7 \\
\hline $\mathrm{C} 15-\mathrm{C} 16-\mathrm{H} 16$ & 119.7 \\
\hline
\end{tabular}

119.8

119.8

$121.1(4)$

119.5

119.5

118.0 (4)

120.7 (4)

$121.2(4)$

$121.2(4)$

119.4

119.4

119.6 (4)

120.2

120.2

109.5

109.5

109.5

109.5

109.5

109.5

111.57 (19)

$113.15(19)$

$111.8(2)$

$107.21(17)$

$106.72(17)$

105.94 (16)

$119.4(4)$

$121.7(3)$

$118.8(4)$

$119.4(5)$

120.3

120.3

$122.0(5)$

119.0

119.0

117.9 (4)

120.7 (5)

$121.5(5)$

120.8 (4)

119.6

119.6

120.5 (4)

119.7

119.7

109.5

109.5

109.5

109.5 


\begin{tabular}{|c|c|}
\hline $\mathrm{C} 22-\mathrm{C} 21-\mathrm{C} 26$ & $117.5(3)$ \\
\hline $\mathrm{C} 22-\mathrm{C} 21-\mathrm{C} 1$ & $125.4(3)$ \\
\hline $\mathrm{C} 26-\mathrm{C} 21-\mathrm{C} 1$ & $117.1(3)$ \\
\hline $\mathrm{C} 23-\mathrm{C} 22-\mathrm{C} 21$ & $121.5(4)$ \\
\hline $\mathrm{C} 23-\mathrm{C} 22-\mathrm{H} 22$ & 119.3 \\
\hline $\mathrm{C} 21-\mathrm{C} 22-\mathrm{H} 22$ & 119.3 \\
\hline $\mathrm{C} 24-\mathrm{C} 23-\mathrm{C} 22$ & $118.3(4)$ \\
\hline $\mathrm{C} 24-\mathrm{C} 23-\mathrm{H} 23$ & 120.8 \\
\hline $\mathrm{C} 22-\mathrm{C} 23-\mathrm{H} 23$ & 120.8 \\
\hline $\mathrm{C} 25-\mathrm{C} 24-\mathrm{C} 23$ & 123.3 \\
\hline $\mathrm{C} 25-\mathrm{C} 24-\mathrm{F} 24$ & $118.8(4)$ \\
\hline $\mathrm{C} 23-\mathrm{C} 24-\mathrm{F} 24$ & $117.9(4)$ \\
\hline $\mathrm{C} 24-\mathrm{C} 25-\mathrm{C} 26$ & $119.0(4)$ \\
\hline $\mathrm{C} 24-\mathrm{C} 25-\mathrm{H} 25$ & 120.5 \\
\hline $\mathrm{C} 26-\mathrm{C} 25-\mathrm{H} 25$ & 120.5 \\
\hline $\mathrm{C} 21-\mathrm{C} 26-\mathrm{C} 25$ & $120.3(4)$ \\
\hline $\mathrm{C} 21-\mathrm{C} 26-\mathrm{H} 26$ & 119.8 \\
\hline $\mathrm{C} 25-\mathrm{C} 26-\mathrm{H} 26$ & 119.8 \\
\hline $\mathrm{C} 42-\mathrm{C} 41-\mathrm{N} 4$ & $114.5(3)$ \\
\hline $\mathrm{C} 42-\mathrm{C} 41-\mathrm{H} 41 \mathrm{~A}$ & 108.6 \\
\hline $\mathrm{N} 4-\mathrm{C} 41-\mathrm{H} 41 \mathrm{~A}$ & 108.6 \\
\hline $\mathrm{C} 42-\mathrm{C} 41-\mathrm{H} 41 \mathrm{~B}$ & 108.6 \\
\hline $\mathrm{N} 4-\mathrm{C} 41-\mathrm{H} 41 \mathrm{~B}$ & 108.6 \\
\hline $\mathrm{H} 41 \mathrm{~A}-\mathrm{C} 41-\mathrm{H} 41 \mathrm{~B}$ & 107.6 \\
\hline $\mathrm{C} 43-\mathrm{C} 42-\mathrm{C} 41$ & $124.6(4)$ \\
\hline $\mathrm{C} 43-\mathrm{C} 42-\mathrm{H} 42$ & 117.7 \\
\hline $\mathrm{C} 41-\mathrm{C} 42-\mathrm{H} 42$ & 117.7 \\
\hline $\mathrm{C} 42-\mathrm{C} 43-\mathrm{C} 31$ & $127.2(4)$ \\
\hline $\mathrm{C} 42-\mathrm{C} 43-\mathrm{H} 43$ & 116.4 \\
\hline $\mathrm{C} 31-\mathrm{C} 43-\mathrm{H} 43$ & 116.4 \\
\hline $\mathrm{C} 36-\mathrm{C} 31-\mathrm{C} 32$ & $118.0(4)$ \\
\hline $\mathrm{C} 36-\mathrm{C} 31-\mathrm{C} 43$ & $124.6(4)$ \\
\hline $\mathrm{C} 32-\mathrm{C} 31-\mathrm{C} 43$ & $117.4(4)$ \\
\hline $\mathrm{C} 33-\mathrm{C} 32-\mathrm{C} 31$ & $121.3(4)$ \\
\hline $\mathrm{C} 33-\mathrm{C} 32-\mathrm{H} 32$ & 119.3 \\
\hline $\mathrm{C} 31-\mathrm{C} 32-\mathrm{H} 32$ & 119.3 \\
\hline $\mathrm{C} 34-\mathrm{C} 33-\mathrm{C} 32$ & $121.2(5)$ \\
\hline $\mathrm{C} 34-\mathrm{C} 33-\mathrm{H} 33$ & 119.4 \\
\hline $\mathrm{C} 32-\mathrm{C} 33-\mathrm{H} 33$ & 119.4 \\
\hline $\mathrm{C} 33-\mathrm{C} 34-\mathrm{C} 35$ & $119.2(4)$ \\
\hline $\mathrm{C} 6-\mathrm{N} 1-\mathrm{C} 2-\mathrm{C} 3$ & $-55.3(4)$ \\
\hline $\mathrm{C} 1-\mathrm{N} 1-\mathrm{C} 2-\mathrm{C} 3$ & $-177.3(3)$ \\
\hline $\mathrm{N} 1-\mathrm{C} 2-\mathrm{C} 3-\mathrm{N} 4$ & $55.4(4)$ \\
\hline $\mathrm{C} 2-\mathrm{C} 3-\mathrm{N} 4-\mathrm{C} 5$ & $-53.2(4)$ \\
\hline $\mathrm{C} 2-\mathrm{C} 3-\mathrm{N} 4-\mathrm{C} 41$ & $-179.7(3)$ \\
\hline $\mathrm{C} 3-\mathrm{N} 4-\mathrm{C} 5-\mathrm{C} 6$ & $54.7(4)$ \\
\hline $\mathrm{C} 41-\mathrm{N} 4-\mathrm{C} 5-\mathrm{C} 6$ & $-177.3(3)$ \\
\hline
\end{tabular}

\begin{tabular}{|c|c|}
\hline $\mathrm{H} 67 \mathrm{~A}-\mathrm{C} 67-\mathrm{H} 67 \mathrm{C}$ & 109.5 \\
\hline $\mathrm{H} 67 \mathrm{~B}-\mathrm{C} 67-\mathrm{H} 67 \mathrm{C}$ & 109.5 \\
\hline $\mathrm{O} 63-\mathrm{S} 61-\mathrm{O} 62$ & $116.4(4)$ \\
\hline $\mathrm{O} 63-\mathrm{S} 61-\mathrm{O} 61$ & $109.1(4)$ \\
\hline $\mathrm{O} 62-\mathrm{S} 61-\mathrm{O} 61$ & $112.5(2)$ \\
\hline O63-S61-C61 & $104.6(3)$ \\
\hline $\mathrm{O} 62-\mathrm{S} 61-\mathrm{C} 61$ & $108.2(2)$ \\
\hline $\mathrm{O} 61-\mathrm{S} 61-\mathrm{C} 61$ & $105.1(2)$ \\
\hline $\mathrm{C} 66 \mathrm{~A}-\mathrm{C} 61 \mathrm{~A}-\mathrm{C} 62 \mathrm{~A}$ & $118.3(7)$ \\
\hline $\mathrm{C} 66 \mathrm{~A}-\mathrm{C} 61 \mathrm{~A}-\mathrm{S} 61 \mathrm{~A}$ & $121.2(7)$ \\
\hline $\mathrm{C} 62 \mathrm{~A}-\mathrm{C} 61 \mathrm{~A}-\mathrm{S} 61 \mathrm{~A}$ & $118.5(8)$ \\
\hline $\mathrm{C} 61 \mathrm{~A}-\mathrm{C} 62 \mathrm{~A}-\mathrm{C} 63 \mathrm{~A}$ & $119.1(9)$ \\
\hline $\mathrm{C} 61 \mathrm{~A}-\mathrm{C} 62 \mathrm{~A}-\mathrm{H} 62 \mathrm{~A}$ & 120.5 \\
\hline $\mathrm{C} 63 \mathrm{~A}-\mathrm{C} 62 \mathrm{~A}-\mathrm{H} 62 \mathrm{~A}$ & 120.5 \\
\hline $\mathrm{C} 64 \mathrm{~A}-\mathrm{C} 63 \mathrm{~A}-\mathrm{C} 62 \mathrm{~A}$ & $121.5(11)$ \\
\hline $\mathrm{C} 64 \mathrm{~A}-\mathrm{C} 63 \mathrm{~A}-\mathrm{H} 63 \mathrm{~A}$ & 119.3 \\
\hline $\mathrm{C} 62 \mathrm{~A}-\mathrm{C} 63 \mathrm{~A}-\mathrm{H} 63 \mathrm{~A}$ & 119.3 \\
\hline $\mathrm{C} 63 \mathrm{~A}-\mathrm{C} 64 \mathrm{~A}-\mathrm{C} 65 \mathrm{~A}$ & $117.1(7)$ \\
\hline $\mathrm{C} 63 \mathrm{~A}-\mathrm{C} 64 \mathrm{~A}-\mathrm{C} 67 \mathrm{~A}$ & $120.7(9)$ \\
\hline $\mathrm{C} 65 \mathrm{~A}-\mathrm{C} 64 \mathrm{~A}-\mathrm{C} 67 \mathrm{~A}$ & $121.2(11)$ \\
\hline $\mathrm{C} 66 \mathrm{~A}-\mathrm{C} 65 \mathrm{~A}-\mathrm{C} 64 \mathrm{~A}$ & $120.4(8)$ \\
\hline $\mathrm{C} 66 \mathrm{~A}-\mathrm{C} 65 \mathrm{~A}-\mathrm{H} 65 \mathrm{~A}$ & 119.8 \\
\hline $\mathrm{C} 64 \mathrm{~A}-\mathrm{C} 65 \mathrm{~A}-\mathrm{H} 65 \mathrm{~A}$ & 119.8 \\
\hline $\mathrm{C} 61 \mathrm{~A}-\mathrm{C} 66 \mathrm{~A}-\mathrm{C} 65 \mathrm{~A}$ & $120.4(7)$ \\
\hline C61A-C66A-H66A & 119.8 \\
\hline C65A-C66A-H66A & 119.8 \\
\hline $\mathrm{C} 64 \mathrm{~A}-\mathrm{C} 67 \mathrm{~A}-\mathrm{H} 67 \mathrm{D}$ & 109.5 \\
\hline $\mathrm{C} 64 \mathrm{~A}-\mathrm{C} 67 \mathrm{~A}-\mathrm{H} 67 \mathrm{E}$ & 109.5 \\
\hline H67D-C67A-H67E & 109.5 \\
\hline $\mathrm{C} 64 \mathrm{~A}-\mathrm{C} 67 \mathrm{~A}-\mathrm{H} 67 \mathrm{~F}$ & 109.5 \\
\hline $\mathrm{H} 67 \mathrm{D}-\mathrm{C} 67 \mathrm{~A}-\mathrm{H} 67 \mathrm{~F}$ & 109.5 \\
\hline $\mathrm{H} 67 \mathrm{E}-\mathrm{C} 67 \mathrm{~A}-\mathrm{H} 67 \mathrm{~F}$ & 109.5 \\
\hline $\mathrm{O} 63 \mathrm{~A}-\mathrm{S} 61 \mathrm{~A}-\mathrm{O} 62 \mathrm{~A}$ & $117.7(7)$ \\
\hline $\mathrm{O} 63 \mathrm{~A}-\mathrm{S} 61 \mathrm{~A}-\mathrm{O} 61 \mathrm{~A}$ & $107.6(6)$ \\
\hline $\mathrm{O} 62 \mathrm{~A}-\mathrm{S} 61 \mathrm{~A}-\mathrm{O} 61 \mathrm{~A}$ & $110.9(6)$ \\
\hline $\mathrm{O} 63 \mathrm{~A}-\mathrm{S} 61 \mathrm{~A}-\mathrm{C} 61 \mathrm{~A}$ & $106.3(6)$ \\
\hline $\mathrm{O} 62 \mathrm{~A}-\mathrm{S} 61 \mathrm{~A}-\mathrm{C} 61 \mathrm{~A}$ & $108.4(6)$ \\
\hline $\mathrm{O} 61 \mathrm{~A}-\mathrm{S} 61 \mathrm{~A}-\mathrm{C} 61 \mathrm{~A}$ & $105.2(6)$ \\
\hline $\mathrm{H} 71 \mathrm{~A}-\mathrm{O} 71-\mathrm{H} 71 \mathrm{~B}$ & 102.4 \\
\hline $\mathrm{H} 81 \mathrm{~B}-\mathrm{O} 81-\mathrm{H} 81 \mathrm{~A}$ & 107.5 \\
\hline $\mathrm{C} 33-\mathrm{C} 34-\mathrm{C} 35-\mathrm{C} 36$ & $-0.8(7)$ \\
\hline $\mathrm{C} 32-\mathrm{C} 31-\mathrm{C} 36-\mathrm{C} 35$ & $0.2(6)$ \\
\hline $\mathrm{C} 43-\mathrm{C} 31-\mathrm{C} 36-\mathrm{C} 35$ & $-179.4(4)$ \\
\hline $\mathrm{C} 34-\mathrm{C} 35-\mathrm{C} 36-\mathrm{C} 31$ & $0.8(7)$ \\
\hline $\mathrm{C} 56-\mathrm{C} 51-\mathrm{C} 52-\mathrm{C} 53$ & $0.4(6)$ \\
\hline $\mathrm{S} 51-\mathrm{C} 51-\mathrm{C} 52-\mathrm{C} 53$ & $-177.5(3)$ \\
\hline $\mathrm{C} 51-\mathrm{C} 52-\mathrm{C} 53-\mathrm{C} 54$ & $0.0(6)$ \\
\hline
\end{tabular}




\begin{tabular}{|c|c|}
\hline $\mathrm{N} 4-\mathrm{C} 5-\mathrm{C} 6-\mathrm{N} 1$ & $-57.2(4)$ \\
\hline $\mathrm{C} 2-\mathrm{N} 1-\mathrm{C} 6-\mathrm{C} 5$ & $55.9(4)$ \\
\hline $\mathrm{C} 1-\mathrm{N} 1-\mathrm{C} 6-\mathrm{C} 5$ & $179.6(3)$ \\
\hline $\mathrm{C} 6-\mathrm{N} 1-\mathrm{C} 1-\mathrm{C} 21$ & $59.7(3)$ \\
\hline $\mathrm{C} 2-\mathrm{N} 1-\mathrm{C} 1-\mathrm{C} 11$ & $-51.9(4)$ \\
\hline $\mathrm{C} 2-\mathrm{N} 1-\mathrm{C} 1-\mathrm{C} 21$ & $-179.0(3)$ \\
\hline $\mathrm{C} 6-\mathrm{N} 1-\mathrm{C} 1-\mathrm{C} 11$ & $-173.2(3)$ \\
\hline $\mathrm{C} 21-\mathrm{C} 1-\mathrm{C} 11-\mathrm{C} 16$ & $46.3(5)$ \\
\hline $\mathrm{N} 1-\mathrm{C} 1-\mathrm{C} 11-\mathrm{C} 16$ & $-79.4(4)$ \\
\hline $\mathrm{C} 21-\mathrm{C} 1-\mathrm{C} 11-\mathrm{C} 12$ & $-132.4(3)$ \\
\hline $\mathrm{N} 1-\mathrm{C} 1-\mathrm{C} 11-\mathrm{C} 12$ & $101.8(4)$ \\
\hline $\mathrm{C} 16-\mathrm{C} 11-\mathrm{C} 12-\mathrm{C} 13$ & $-0.9(6)$ \\
\hline $\mathrm{C} 1-\mathrm{C} 11-\mathrm{C} 12-\mathrm{C} 13$ & $177.9(3)$ \\
\hline $\mathrm{C} 11-\mathrm{C} 12-\mathrm{C} 13-\mathrm{C} 14$ & $-0.2(6)$ \\
\hline $\mathrm{C} 12-\mathrm{C} 13-\mathrm{C} 14-\mathrm{F} 14$ & $-179.0(4)$ \\
\hline $\mathrm{C} 12-\mathrm{C} 13-\mathrm{C} 14-\mathrm{C} 15$ & $1.6(6)$ \\
\hline $\mathrm{C} 13-\mathrm{C} 14-\mathrm{C} 15-\mathrm{C} 16$ & $-2.0(6)$ \\
\hline $\mathrm{F} 14-\mathrm{C} 14-\mathrm{C} 15-\mathrm{C} 16$ & $178.7(4)$ \\
\hline $\mathrm{C} 12-\mathrm{C} 11-\mathrm{C} 16-\mathrm{C} 15$ & $0.5(6)$ \\
\hline $\mathrm{C} 1-\mathrm{C} 11-\mathrm{C} 16-\mathrm{C} 15$ & $-178.2(3)$ \\
\hline $\mathrm{C} 14-\mathrm{C} 15-\mathrm{C} 16-\mathrm{C} 11$ & $0.8(6)$ \\
\hline $\mathrm{C} 11-\mathrm{C} 1-\mathrm{C} 21-\mathrm{C} 22$ & $-82.7(4)$ \\
\hline $\mathrm{N} 1-\mathrm{C} 1-\mathrm{C} 21-\mathrm{C} 22$ & $42.4(5)$ \\
\hline $\mathrm{C} 11-\mathrm{C} 1-\mathrm{C} 21-\mathrm{C} 26$ & $97.7(4)$ \\
\hline $\mathrm{N} 1-\mathrm{C} 1-\mathrm{C} 21-\mathrm{C} 26$ & $-137.2(3)$ \\
\hline $\mathrm{C} 26-\mathrm{C} 21-\mathrm{C} 22-\mathrm{C} 23$ & $-0.8(6)$ \\
\hline $\mathrm{C} 1-\mathrm{C} 21-\mathrm{C} 22-\mathrm{C} 23$ & $179.6(4)$ \\
\hline $\mathrm{C} 21-\mathrm{C} 22-\mathrm{C} 23-\mathrm{C} 24$ & $-0.4(7)$ \\
\hline $\mathrm{C} 22-\mathrm{C} 23-\mathrm{C} 24-\mathrm{C} 25$ & $0.5(7)$ \\
\hline $\mathrm{C} 22-\mathrm{C} 23-\mathrm{C} 24-\mathrm{F} 24$ & $179.7(4)$ \\
\hline $\mathrm{C} 23-\mathrm{C} 24-\mathrm{C} 25-\mathrm{C} 26$ & $0.5(7)$ \\
\hline $\mathrm{F} 24-\mathrm{C} 24-\mathrm{C} 25-\mathrm{C} 26$ & $-178.7(4)$ \\
\hline $\mathrm{C} 22-\mathrm{C} 21-\mathrm{C} 26-\mathrm{C} 25$ & $1.9(6)$ \\
\hline $\mathrm{C} 1-\mathrm{C} 21-\mathrm{C} 26-\mathrm{C} 25$ & $-178.5(4)$ \\
\hline $\mathrm{C} 24-\mathrm{C} 25-\mathrm{C} 26-\mathrm{C} 21$ & $-1.7(7)$ \\
\hline $\mathrm{C} 3-\mathrm{N} 4-\mathrm{C} 41-\mathrm{C} 42$ & $-56.2(4)$ \\
\hline $\mathrm{C} 5-\mathrm{N} 4-\mathrm{C} 41-\mathrm{C} 42$ & $178.0(3)$ \\
\hline $\mathrm{N} 4-\mathrm{C} 41-\mathrm{C} 42-\mathrm{C} 43$ & $121.6(4)$ \\
\hline $\mathrm{C} 41-\mathrm{C} 42-\mathrm{C} 43-\mathrm{C} 31$ & $172.9(3)$ \\
\hline $\mathrm{C} 42-\mathrm{C} 43-\mathrm{C} 31-\mathrm{C} 36$ & $12.6(6)$ \\
\hline $\mathrm{C} 42-\mathrm{C} 43-\mathrm{C} 31-\mathrm{C} 32$ & $-167.1(4)$ \\
\hline $\mathrm{C} 36-\mathrm{C} 31-\mathrm{C} 32-\mathrm{C} 33$ & $-1.3(6)$ \\
\hline $\mathrm{C} 43-\mathrm{C} 31-\mathrm{C} 32-\mathrm{C} 33$ & $178.3(4)$ \\
\hline $\mathrm{C} 31-\mathrm{C} 32-\mathrm{C} 33-\mathrm{C} 34$ & $1.4(7)$ \\
\hline $\mathrm{C} 32-\mathrm{C} 33-\mathrm{C} 34-\mathrm{C} 35$ & $-0.3(7)$ \\
\hline
\end{tabular}

$-0.6(6)$

$177.9(4)$

$0.6(6)$

-177.8 (4)

-0.4 (6)

177.5 (3)

-0.2 (6)

73.3 (3)

$-104.6(3)$

-46.4 (3)

135.8 (3)

$-165.6(3)$

16.5 (4)

2.5 (17)

-173.3 (9)

-3.3 (18)

1.7 (15)

-178.7 (13)

0.7 (13)

-178.9 (9)

-0.2 (11)

175.5 (4)

-1.4 (9)

$-58.5(5)$

$117.3(10)$

176.8 (4)

$-7.5(10)$

56.4 (4)

$-127.8(9)$

-8 (7)

157 (4)

18 (7)

$-12(6)$

157 (7)

-5 (7)

$-174(5)$

$-9(5)$

$-173(3)$

16 (6)

-95 (2)

101 (4)

137 (2)

-27 (4)

19 (2)

-145 (4) 


\section{supporting information}

Hydrogen-bond geometry $\left(\AA,{ }^{\circ}\right)$

\begin{tabular}{lllll}
\hline$D-\mathrm{H} \cdots A$ & $D-\mathrm{H}$ & $\mathrm{H} \cdots A$ & $D \cdots A$ & $D-\mathrm{H} \cdots A$ \\
\hline $\mathrm{N} 1-\mathrm{H} 1 \cdots \mathrm{O} 71$ & 0.93 & 1.79 & $2.716(4)$ & 169 \\
$\mathrm{~N} 4-\mathrm{H} 4 \cdots \mathrm{O} 81$ & 1.00 & 1.69 & $2.690(4)$ & 173 \\
$\mathrm{O} 71-\mathrm{H} 71 A \cdots \mathrm{O} 51$ & 0.85 & 1.88 & $2.708(4)$ & 167 \\
$\mathrm{O} 71-\mathrm{H} 71 B \cdots \mathrm{O} 61^{\mathrm{i}}$ & 0.84 & 1.87 & $2.675(5)$ & 160 \\
$\mathrm{O} 81-\mathrm{H} 81 A \cdots \mathrm{O} 61$ & 0.84 & 2.05 & $2.852(5)$ & 161 \\
$\mathrm{O} 81-\mathrm{H} 81 B \cdots \mathrm{O} 52^{\mathrm{ii}}$ & 0.84 & 1.92 & $2.748(4)$ & 167 \\
$\mathrm{C} 1-\mathrm{H} 1 A \cdots \mathrm{O} 63$ & 1.00 & 2.23 & $3.139(7)$ & 151 \\
$\mathrm{C} 2-\mathrm{H} 2 B \cdots \mathrm{O} 63$ & 0.99 & 2.47 & $3.314(7)$ & 143 \\
$\mathrm{C} 6-\mathrm{H} 6 A \cdots \mathrm{O} 53^{\mathrm{iii}}$ & 0.99 & 2.37 & $3.191(5)$ & 140 \\
$\mathrm{C} 16-\mathrm{H} 16 \cdots \mathrm{O} 62^{\mathrm{i}}$ & 0.95 & 2.41 & $3.228(6)$ & 144 \\
$\mathrm{C} 22-\mathrm{H} 22 \cdots \mathrm{O} 71$ & 0.95 & 2.49 & $3.381(5)$ & 156 \\
$\mathrm{C} 34-\mathrm{H} 34 \cdots \mathrm{O} 51^{\mathrm{iv}}$ & 0.95 & 2.42 & $3.362(6)$ & 169 \\
$\mathrm{C} 41-\mathrm{H} 41 A \cdots \mathrm{O} 53$ & 0.99 & 2.35 & $3.280(5)$ & 157 \\
$\mathrm{C} 41-\mathrm{H} 41 B \cdots \mathrm{O} 61^{\mathrm{v}}$ & 0.99 & 2.37 & $3.341(5)$ & 167
\end{tabular}

Symmetry codes: (i) $x+1, y, z$; (ii) $x-1, y, z$; (iii) $-x+2,-y+1,-z$; (iv) $-x+2, y+1 / 2,-z+1 / 2$; (v) $-x+1,-y+1,-z$. 